\title{
ROBUSTNESS OF FREDHOLM PROPERTIES OF PARABOLIC EVOLUTION EQUATIONS UNDER BOUNDARY PERTURBATIONS
}

\author{
LAHCEN MANIAR AND ROLAND SCHNAUBELT
}

\begin{abstract}
We study perturbations at the boundary of linear nonautonomous parabolic boundary value problems. Our approach relies on a transformation of the given inhomogeneous boundary value problem to an evolution equation in larger, time varying extrapolation spaces. We establish the well-posedness of this equation and Duhamel's formulas relating the evolution families solving the perturbed and the unperturbed problem. By means of these formulas, we can show that the perturbed evolution equation inherits the exponential dichotomy and Fredholm properties of the unperturbed one if the perburbations are small in norm or compact. This result leads to a Fredholm alternative for the given perturbed boundary value problem.
\end{abstract}

\section{INTRODUCTION}

We study perturbations at the boundary of linear nonautonomous parabolic boundary value problems. Such perturbations occur for instance if a feedback mechanism is applied at the boundary of a system governed by a parabolic differential equation. Our main results show that the perturbed problem inherits the well-posedness and the long term behaviour of the unperturbed system under suitable assumptions on the perturbations. Our interest is focussed on the exponential dichotomy of the homogeneous equation and on Fredholm properties of the inhomogeneous problem on the time interval $\mathbb{R}$. Namely, we establish an Fredholm alternative for the perturbed problem with inhomogeneities at the boundary.

Following a common approach in, e.g., control theory (see [1] and the references in [16]), we transform the given perturbed boundary value problem into the evolution equation

$$
u^{\prime}(t)=A_{-1}(t) u(t)+B(t) u(t)+f(t), \quad t \in \mathbb{R},
$$

see Section 5. Here $A(t), t \in \mathbb{R}$, are sectorial operators in a Banach space $X, \hat{X}^{t}$ is the closure of the domain $D(A(t))$ in $X$, and $A_{-1}(t): \hat{X}^{t} \rightarrow X_{-1}^{t}$ is the extension of $A(t)$ in the extrapolation space $X_{-1}^{t}$ which is the completion of $\hat{X}^{t}$ with respect to the norm $\left\|(\omega-A(t))^{-1} x\right\|$, for some $\omega \in \rho(A(t))$. In particular, (1.1) is an equation in $X_{-1}^{t}$. The operators $B(t)$ (resp., the function $f$ ) contain the extrapolated operators $A_{-1}(t)$, the solution operator of the elliptic boundary value problem associated with $A(t)$ and the given perturbation (resp., the given inhomogeneity) at the boundary; see Section 5 .

In our main analysis we abstract from this special structure and consider perturbations $B(t)$ which, for some $\alpha \in(0,1)$, map the continuous interpolation space $(X, D(A(t)))_{\alpha}=$ : $X_{\alpha}^{t}$ to $\left(X_{-1}^{t}, \hat{X}^{t}\right)_{\alpha}=: X_{\alpha-1}^{t}$. (These concepts are recalled in Section 2.) Our crucial

2000 Mathematics Subject Classification. 35K20, 35K90, 47A53, 47A55, 47D06.

Key words and phrases. Fredholm operator, index, exponential dichotomy, robustness, inhomogeneous evolution equation, evolution family, parabolic initial-boundary value problem, inter- and extrapolation.

This work was supported by the DFG grant 445 MAR-113/10/0-2. L.M. gratefully acknowledges the support by the Alexander-von-Humboldt foundation. 
assumption says that the norm of $R\left(\bar{\omega}, A_{-1}(t)\right) B(t)$ in $\mathcal{L}\left(X_{\alpha}^{t}\right)$ is smaller than an explicitely given constant for a sufficiently large $\bar{\omega} \geq 0$ and for each $t \in \mathbb{R}$. The case $\alpha=1$ (i.e, perturbations $B(t): D(A(t)) \rightarrow X$ ) was already treated in [7] in a somewhat more special setting, so that we exclude it from our investigations for simplicity. We further assume that the sectorial operators $A(t)$ satisfy the so-called Acquistapace-Terreni conditions (2.1) and (2.2). These conditions are quite flexible in so far they only require a Hölder condition in $t$ and they allow for non-dense and time varying domains $D(A(t))$. Under these conditions the family $A(\cdot)$ generates an evolution family $U(\cdot, \cdot)$ on $X$ having parabolic regularity, as described in Section 2. Linear elliptic partial differential operators with time varying coefficients and boundary conditions typically lead to operators $A(t)$ satisfying (2.1) and (2.2), cf. Example 5.9.

Since we are mainly interested in the asymptotic behaviour, we only look for solutions of (1.1) in an integrated sense, as introduced at the end of Section 3. But we note that due to Proposition 2.6 of [16] such mild solutions actually solve (1.1) pointwise in the spaces $X_{\beta-1}^{t}$ for $\beta<\alpha$. (See also $[4, \S 5.2]$ for the case of time independent $X_{\alpha}^{t}=X_{\alpha}$ and $X_{\alpha-1}^{t}=X_{\alpha-1}$.) Our analysis relies on Proposition 3.6 which states that the parts $C(t)$ of $A_{-1}(t)+B(t)$ in $X$ again satisfy the Aquistapace-Terreni conditions, and thus generate an evolution family $U_{B}(\cdot, \cdot)$ on $X$ having parabolic regularity. In particular there exist the corresponding inter/extrapolation spaces $X_{\alpha}^{C(t)}$ and $X_{\alpha-1}^{C(t)}$. Moreover, in Theorem 3.7 we establish Duhamel's formulas

$$
\begin{aligned}
& U_{B}(t, s) x=U(t, s) x+\int_{s}^{t} U_{\alpha-1}(t, \tau) B(\tau) U_{B}(\tau, s) x d \tau, \\
& U_{B}(t, s) x=U(t, s) x+\int_{s}^{t} U_{B, \alpha-1}(t, \tau) B(\tau) U(\tau, s) x d \tau,
\end{aligned}
$$

$x \in X$ and $t \geq s$. Here the subscript ' $\alpha-1$ ' denotes the extensions of the evolution families to the spaces $X_{\alpha-1}^{t}$ and $X_{\alpha-1}^{C(t)}$, respectively, which exist because of the Aquistapace-Terreni conditions, see Proposition 2.1. Of course, these formulas only make sense if embeddings such as $X_{\alpha+\epsilon}^{C(t)} \hookrightarrow X_{\alpha}^{t}$ and $X_{\alpha-1}^{t} \hookrightarrow X_{\alpha-1}^{C(t)}$ hold. These embeddings are in fact established in Lemma 3.3. In Proposition 3.4 we even prove that $X_{\alpha-j}^{t}$ and $X_{\alpha-j}^{C(t)}$ are isomorphic $(j=0,1)$ under somewhat stronger conditions on $B(t)$. In all these results it is crucial to check that the resulting constants do not depend on $t$. We note that the sectoriality of $C(t)$ was shown in [12] in a slightly different setting. In [10] the isomorphy of $X_{\beta}^{t}$ and $X_{\beta}^{C(t)}$ for $\beta \in(\alpha-1, \alpha)$ was verified in a more general situation. To our knowledge there are no corresponding perturbations results concerning the Aquistapace-Terreni conditions, and also the equations (1.2) were not known before.

Based on the formulas (1.2) and the regularity properties of $U(\cdot, \cdot)$ and $U_{B}(\cdot, \cdot)$, we can then derive our main results concerning the asymptotic behaviour of (1.1) in Section 4. If the operators $B(t)$ have sufficiently small norms in $\mathcal{L}\left(X_{\alpha}^{t}, X_{\alpha-1}^{t}\right)$, then $U_{B}(\cdot, \cdot)$ inherits the exponential dichotomy of $U(\cdot, \cdot)$ due to Theorem 4.1. Moreover, the Fredholmity of the full equation (1.1) follows from the Fredholmity of (1.1) with $B(t)=0$ by Theorem 4.2 , and this implication is also valid if the operators $B(t)$ are compact and vanish at infinity, see Theorem 4.3. These facts lead to a Fredholm alternative for (1.1) stated in Theorem 4.5. In Section 4 we use the Fredholm theory developed in [13], [14] and [16]. For further information on this subject we refer to the references therein and [6], [7]. In [16] we have studied the Fredholm properties of (1.1) for the case $B(t)=0$ in detail. It turns out that 
(1.1) with $B(t)=0$ is Fredholm if the resolvents $(\omega-A(t))^{-1}$ converge in norm to the resolvents $\left(\omega-A_{ \pm \infty}\right)^{-1}$ as $t \rightarrow \pm \infty$, where $A_{ \pm \infty}$ are sectorial operators with compactly embedded domains and without spectrum on $i \mathbb{R}$, cf. Example 5.9 and [16, Corollary 3.7]. In the last section we then translate our main results from Section 4 into the setting of our motivating application to boundary perturbations.

Notation. We denote by $D(A), N(A), R(A), \sigma(A), \rho(A)$ the domain, kernel, image, spectrum and resolvent set of a linear operator $A$, and we set $R(\lambda, A):=(\lambda I-A)^{-1}=$ $(\lambda-A)^{-1}$ for $\lambda \in \rho(A)$. Moreover, $\mathcal{L}(X, Y)$ is the space of bounded linear operators between two Banach spaces $X$ and $Y$, where $\mathcal{L}(X, X)=\mathcal{L}(X)$. For $\phi \in(0, \pi]$ and $\omega \in \mathbb{R}$, we define $\Sigma_{\phi, \omega}=\{\lambda \in \mathbb{C} \backslash\{\omega\}:|\arg (\lambda-\omega)|<\phi\}$ and $\Sigma_{\phi}:=\Sigma_{\phi, 0}$. By $c(\alpha, \cdots)$ we designate a generic constant depending on quantities $\alpha, \cdots$.

\section{Evolution families AND EXTRAPOlation}

We investigate linear operators $A(t), t \in \mathbb{R}$, on a Banach space $X$ subject to the following hypotheses introduced by P. Acquistapace and B. Terreni in [2] and [3]. There are constants $\omega \in \mathbb{R}, \phi \in(\pi / 2, \pi), K, L>0$ and $\mu, \nu \in(0,1]$ such that $\mu+\nu>1$ and

$$
\begin{aligned}
& \Sigma_{\phi, \omega} \subset \rho(A(t)), \quad\|R(\lambda, A(t))\| \leq \frac{K}{1+|\lambda-\omega|}, \\
& \|(A(t)-\omega) R(\lambda, A(t))[R(\omega, A(t))-R(\omega, A(s))]\| \leq L \frac{|t-s|^{\mu}}{|\lambda-\omega|^{\nu}}
\end{aligned}
$$

for all $t \in \mathbb{R}$ and $\lambda \in \Sigma_{\phi, \omega}$. Observe that the domains $D(A(t))$ are not required to be dense. These conditions imply that the operators $A(\cdot)$ generate a unique evolution family $U(t, s)$ of parabolic type. This means that $U(t, s) U(s, r)=U(t, r)$ and $U(t, t)=I$ for $t \geq s \geq r$ in $\mathbb{R}$, that the map $(t, s) \mapsto U(t, s) \in \mathcal{L}(X)$ is continuous for $t>s$, and that $t \mapsto U(t, s) \in \mathcal{L}(X)$ is continuously differentiable, $U(t, s) X \subseteq D(A(t))$, and $\partial_{t} U(t, s)=A(t) U(t, s)$ for $t>s$. Moreover, for $s \in \mathbb{R}$ and $x \in \overline{D(A(s))}$, the function $t \mapsto u(t)=U(t, s) x$ is continuous at $t=s$ and $u$ is the unique solution in $C([s, \infty), X) \cap C^{1}((s, \infty), X)$ of the Cauchy problem

$$
u^{\prime}(t)=A(t) u(t), \quad t>s, \quad u(s)=x .
$$

Finally, we have $U(t, s) x \rightarrow x$ as $s \nearrow t$ if $x \in \overline{D(A(t))}$. These facts have been established in [2] and [3], see also [1], [4], [15], [19], [20].

Before stating further regularity properties of $U(t, s)$, we have to introduce the inter- and extrapolation spaces for $A(t)$. We refer to [4], [8], and [15] for proofs and more details. Let $A$ be a sectorial operator on $X$ (i.e., (2.1) holds with $A(t)$ replaced by $A$ ) and $\alpha \in(0,1)$. We define the new norm on $D(A)$ by

$$
\|x\|_{\alpha}^{A}:=\sup _{r>0}\left\|r^{\alpha}(A-\omega) R(r, A-\omega) x\right\|,
$$

and consider the continuous interpolation spaces $X_{\alpha}^{A}:=\overline{D(A)}\|\cdot\|_{\alpha}^{A}$ which are Banach spaces endowed with the norms $\|\cdot\|_{\alpha}^{A}$. For convenience we further write $X_{0}^{A}:=X,\|x\|_{0}^{A}:=\|x\|$, $X_{1}^{A}:=D(A)$ and $\|x\|_{1}^{A}:=\|(\omega-A) x\|$. We also need the closed subspace $\hat{X}^{A}:=\overline{D(A)}$ of $X$. Moreover, we define the extrapolation space $X_{-1}^{A}$ as the completion of $\hat{X}^{A}$ with respect to the norm $\|x\|_{-1}^{A}:=\|R(\omega, A) x\|$. Then $A$ has a unique continuous extension $A_{-1}: \hat{X}^{A} \rightarrow X_{-1}^{A}$. The operator $A_{-1}$ satisfies (2.1) in $X_{-1}^{A}$, it is densely defined, it has the same spectrum as $A$, and it generates the analytic semigroup $e^{t A_{-1}}$ on $X_{-1}^{A}$ being the 
extension of $e^{t A}$. As above, we can then define the space

$$
X_{\alpha-1}^{A}:=\left(X_{-1}\right)_{\alpha}^{A_{-1}} \quad \text { with the norm }\|x\|_{\alpha-1}^{A}:=\|x\|_{\alpha}^{A_{-1}}=\sup _{r>0}\left\|r^{\alpha} R\left(r, A_{-1}-\omega\right) x\right\| .
$$

The restriction $A_{\alpha-1}: X_{\alpha}^{A} \rightarrow X_{\alpha-1}^{A}$ of $A_{-1}$ is sectorial in $X_{\alpha-1}^{A}$ with the same constants as $A$, it has the same spectrum as $A$, and the analytic semigroup $e^{t A_{\alpha-1}}$ on $X_{\alpha-1}^{A}$ is the extension of $e^{t A}$. Observe that $\omega-A_{\alpha-1}: X_{\alpha}^{A} \rightarrow X_{\alpha-1}^{A}$ is an isometric isomorphism. We will frequently use the continuous embeddings

$$
\begin{aligned}
& D(A) \hookrightarrow X_{\beta}^{A} \hookrightarrow D\left((\omega-A)^{\alpha}\right) \hookrightarrow X_{\alpha}^{A} \hookrightarrow \hat{X}^{A} \subset X, \\
& X \hookrightarrow X_{\beta-1}^{A} \hookrightarrow D\left(\left(\omega-A_{-1}\right)^{\alpha}\right) \hookrightarrow X_{\alpha-1}^{A} \hookrightarrow X_{-1}^{A}
\end{aligned}
$$

for all $0<\alpha<\beta<1$, where the fractional powers are defined as usually, cf. [4]. Using the above definitions, one easily deduces the estimates

$$
\begin{aligned}
\left\|R\left(\lambda, A_{-1}\right)\right\|_{\mathcal{L}\left(X_{\alpha}^{A}\right)} & \leq \frac{K}{1+|\lambda-\omega|}, \\
\left\|R\left(\lambda, A_{-1}\right)\right\|_{\mathcal{L}\left(X_{\alpha-1}^{A}, X_{\alpha}^{A}\right)} & \leq 1+K, \\
\left\|R\left(\lambda, A_{-1}\right)\right\|_{\mathcal{L}\left(X_{\alpha-1}^{A}\right)} & \leq \frac{K}{1+|\lambda-\omega|}, \\
\|R(\lambda, A)\|_{\mathcal{L}\left(X, X_{\alpha}^{A}\right)} & \leq \bar{c}(1+|\lambda-\omega|)^{\alpha-1}, \\
\left\|R\left(\lambda, A_{-1}\right)\right\|_{\mathcal{L}\left(X_{\alpha-1}^{A}, X_{\beta}^{A}\right)} & \leq \bar{c}(1+|\lambda-\omega|)^{\beta-\alpha},
\end{aligned}
$$

for every $\lambda \in \Sigma_{\phi, \omega}$ and $0 \leq \beta \leq \alpha \leq 1$, and some constant $\bar{c}$ only depending on $K$.

Given operators $A(t), t \in \mathbb{R}$, satisfying (2.1), we set $X_{\alpha}^{t}=X_{\alpha}^{A(t)}, X_{\alpha-1}^{t}=X_{\alpha-1}^{A(t)}$, and $\hat{X}^{t}=\hat{X}^{A(t)}$ for $0 \leq \alpha \leq 1$ and $t \in \mathbb{R}$, and denote the corresponding norms by $\|x\|_{\alpha-j}^{t}$. Then the norms of the embeddings in (2.3) and the constants in the estimates (2.4)-(2.8) (replacing $A$ by $A(t)$ ) do not depend on $t \in \mathbb{R}$. Let $J \subset \mathbb{R}$ be a closed interval containing more than a point. We further define on $E=E(J):=C_{0}(J, X)$ (the space of continuous functions, vanishing at infinity if $J$ is unbounded) the multiplication operator $A(\cdot)$ by

$$
(A(\cdot) f)(t):=A(t) f(t) \text { for } t \in J, \quad D(A(\cdot)):=\{f \in E: f(t) \in D(A(t)), A(\cdot) f \in E\} .
$$

It is clear that the operator $A(\cdot)$ is also sectorial. We can thus introduce the spaces

$$
E_{\alpha}:=E_{\alpha}^{A(\cdot)}, \quad E_{\alpha-1}:=E_{\alpha-1}^{A(\cdot)}, \quad \text { and } \quad \hat{E}:=\overline{D(A(\cdot))}
$$

for $\alpha \in[0,1]$, where $E_{0}:=E$ and $E_{1}:=D(A(\cdot))$. We observe that $E_{-1} \subseteq \prod_{t \in J} X_{-1}^{t}$ and that the extrapolated operator $A(\cdot)_{-1}: \hat{E} \longrightarrow E_{-1}$ is given by $\left(A(\cdot)_{-1} f\right)(t)=A_{-1}(t) f(t)$ for $t \in J$ and $f \in E$. Further, $E_{\alpha-1}$ has the norm

$$
\|f\|_{\alpha-1}:=\sup _{r>0} \sup _{s \in J}\left\|r^{\alpha} R\left(r, A_{-1}(s)-\omega\right) f(s)\right\| .
$$

Let (2.1) and (2.2) hold. Then there exists a constant $C=C\left(t_{0}\right)>0$ such that

$$
\begin{aligned}
\left\|(\omega-A(t))^{\alpha} e^{\tau A(t)}\right\| & \leq C \tau^{-\alpha}, \\
\left\|U(t, s)(\omega-A(s))^{\theta} y\right\| & \leq C(\mu-\theta)^{-1}(t-s)^{-\theta}\|y\|, \\
\left\|(\omega-A(s))^{\gamma}(R(\omega, A(s))-R(\omega, A(t)))\right\| & \leq C(t-s)^{\mu}, \\
\left\|(\omega-A(t))^{\gamma-1}-(\omega-A(s))^{\gamma-1}\right\| & \leq C(t-s)^{\mu}
\end{aligned}
$$

for all $t, s \in \mathbb{R}$ and $t_{0}>0$ with $0<t-s \leq t_{0}$ and all $0<\tau \leq t_{0}, 0 \leq \alpha \leq 1,0 \leq \theta<\mu$, $0 \leq \gamma<\nu$, and $y \in D\left((\omega-A(s))^{\theta}\right)$. Here, (2.9) is well known and (2.10) was proved in 
[20, Thm.2.1] in a slightly different setting, but the proof also works under the present assumptions. Finally, (2.11) and (2.12) are straightforward consequences of (2.1) and (2.2), cf. [18] and [19]. We state the following result which is crucial for our work. The assertions (i)-(iii) follow from Proposition 2.1 in [16].

Proposition 2.1. Assume that (2.1) and (2.2) hold and let $1-\mu<\alpha<1$ and $0 \leq \beta \leq 1$. Then the following assertions hold for $s<t \leq s+t_{0}$ and $t_{0}>0$ with constants possibly depending on $t_{0}$.

(i) The operators $U(t, s)$ have continuous extensions $U_{\alpha-1}(t, s): X_{\alpha-1}^{s} \rightarrow X$ satisfying

$$
\left\|U_{\alpha-1}(t, s)\right\|_{\mathcal{L}\left(X_{\alpha-1}^{s}, X\right)} \leq c(\alpha)(t-s)^{\alpha-1},
$$

and $U_{\alpha-1}(t, s) x=U_{\gamma-1}(t, s) x$ for $1-\mu<\gamma<\alpha<1$ and $x \in X_{\alpha-1}^{s}$.

(ii) The map $\{(t, s): t>s\} \ni(t, s) \longmapsto U_{\alpha-1}(t, s) f(s) \in X$ is continuous for $f \in E_{\alpha-1}$.

(iii) For $x \in X_{\alpha-1}^{s}$ we have

$$
\left\|U_{\alpha-1}(t, s) x\right\|_{\beta}^{t} \leq c(\alpha)(t-s)^{\alpha-\beta-1}\|x\|_{\alpha-1}^{s} .
$$

(iv) For $x \in X_{\alpha}^{s}$, we have

$$
\frac{\partial^{+}}{\partial s} U(t, s) x=-U_{\alpha-1}(t, s) A_{\alpha-1}(s) x \quad(\text { in } X) .
$$

Proof. (iv) By rescaling we may assume that conditions (2.1) and (2.2) hold with $\omega=0$. Fix $\beta \in(1-\mu, \nu), t>s, h_{0} \in(0, t-s)$, and $x \in X_{\alpha}^{s} \hookrightarrow D\left((-A(s))^{\beta}\right)$, where $\beta<\alpha$. Take $h \in\left[0, h_{0}\right]$. Then $t>h+s$. We use the identities

$$
\begin{aligned}
& U(t, s+h) x-U(t, s) x \\
& =U(t, s+h)(-A(s+h))^{1-\beta}(-A(s+h))^{\beta-1}(x-U(s+h, s) x) \\
& =U_{\beta-1}(t, s+h)\left(-A_{-1}(s+h)\right)^{1-\beta}\left((-A(s))^{\beta-1}-(-A(s+h))^{\beta-1}\right) \int_{0}^{h} A(s) e^{\tau A(s)} x d \tau \\
& \quad+U_{\beta-1}(t, s+h)\left(-A_{-1}(s+h)\right)^{1-\beta}\left(I-e^{h A(s)}\right)(-A(s))^{\beta-1} x \\
& \quad+U_{\beta-1}(t, s+h)\left(-A_{-1}(s+h)\right)^{1-\beta} \int_{s}^{s+h}(-A(s+h))^{\beta-1} U(s+h, \tau)(-A(\tau))^{1-\beta} \\
& \quad \cdot(-A(\tau))^{\beta}[R(0, A(s))-R(0, A(\tau))](-A(s))^{1-\beta} e^{(\tau-s) A(s)}(-A(s))^{\beta} x d \tau \\
& =: S_{1}+S_{2}+S_{3} .
\end{aligned}
$$

It is not difficult to check these equalities for the Yosida approximations $A_{n}(t)$ and the evolution family $U_{n}(\cdot, \cdot)$ generated by $A_{n}(\cdot)$. The above formula then follows by letting $n \rightarrow \infty$ and using [19, Prop.2.1] and the proofs of [16, Lem.5.1] and [19, Prop.3.1]. We now derive that

$$
\begin{aligned}
& \left\|\frac{1}{h} S_{1}\right\| \leq c h^{-1} h^{\mu} h^{\beta} \longrightarrow 0, \quad \frac{1}{h} S_{2} \longrightarrow-U_{\alpha-1}(t, s) A_{\alpha-1}(s) x, \\
& \left\|\frac{1}{h} S_{3}\right\| \leq c h^{-1} \int_{s}^{s+h}(\tau-s)^{\mu}(\tau-s)^{\beta-1} d \tau=c h^{\mu+\beta-1} \longrightarrow 0
\end{aligned}
$$

as $h \rightarrow 0$ by means of (2.9)-(2.12) and [16, Lemma 5.1]. (Note that the constants may depend on $t-h_{0}-s$, but not on $h$.)

Exponential dichotomies are another important tool in our study, cf. [5], [15], [17], [18]. We recall that an evolution family $U(\cdot, \cdot)$ is said to have an exponential dichotomy in an 
interval $J \subset \mathbb{R}$ if there exists a family of projections $P(t) \in \mathcal{L}(X), t \in J$, being strongly continuous with respect to $t$, and numbers $\delta, N>0$ such that

(a) $U(t, s) P(s)=P(t) U(t, s)$,

(b) $U(t, s): Q(s)(X) \rightarrow Q(t)(X)$ is invertible with inverse $\widetilde{U}(s, t)$,

(c) $\|U(t, s) P(s)\| \leq N e^{-\delta(t-s)}$,

(d) $\|\widetilde{U}(s, t) Q(t)\| \leq N e^{-\delta(t-s)}$,

for all $s, t \in J$ with $s \leq t$, where $Q(t):=I-P(t)$ is the 'unstable projection'.

\section{Perturbation Results}

We investigate the perturbed evolution equation

$$
u^{\prime}(t)=A_{-1}(t) u(t)+B(t) u(t), \quad t \in \mathbb{R},
$$

where the operators $A(t), t \in \mathbb{R}$, satisfy the conditions (2.1) and (2.2) on a Banach space $X$ and $B(t): X_{\alpha}^{A(t)} \longrightarrow X_{\alpha-1}^{A(t)}, t \in \mathbb{R}$, are bounded linear perturbations for some $\alpha \in(0,1)$. Then $A(\cdot)$ generates an evolution family $U(\cdot, \cdot)$ on $X$, as recalled in the previous section. Under certain additional hypotheses, we show that the parts $C(t)$ of $A_{\alpha-1}(t)+B(t)$ in $X$ also satisfy the conditions $(2.1)$ and $(2.2)$, and hence generate an evolution family $U_{B}(\cdot, \cdot)$ on $X$. Our main purpose is to show Duhamel's formulas $(1.2)$ for $U_{B}(\cdot, \cdot)$ which will be the key for our study of the asymptotic behavior of $U_{B}(\cdot, \cdot)$ in the next section.

We start with preliminary results about the persistence of inter/extrapolation spaces under perturbations, working in the following setting.

(H1) Let $A$ be a sectorial operator with the constants $\omega=0, K \geq 0$, and $\phi \in(\pi / 2, \pi)$. Let $B \in \mathcal{L}\left(X_{\alpha}^{A}, X_{\alpha-1}^{A}\right)$ for some $\alpha \in(0,1)$. Set $q:=\frac{1}{2 c_{0}}$ and $c_{0}:=1+\frac{K}{\sin (\pi-\phi)}$. We assume that there is an $\bar{\omega} \geq 0$ such that one of the following two conditions hold.

(a) $\left\|R\left(\bar{\omega}, A_{\alpha-1}\right) B\right\|_{\mathcal{L}\left(X_{\alpha}^{A}\right)} \leq q$.

(b) $\left\|B R\left(\bar{\omega}, A_{\alpha-1}\right)\right\|_{\mathcal{L}\left(X_{\alpha-1}^{A}\right)} \leq q$.

Remark 3.1. Hypothesis (H1) holds for the following classes of perturbations $B$ of a sectorial operator $A$ with constants $\omega=0$ and $K>0$. Here (H1)(a) is satisfied in the cases (A), (B) and (D), and (H1)(b) in the cases (B) and (C).

(A) $B: X_{\alpha}^{A} \longrightarrow X_{\alpha-1}^{A}$ is a compact operator. Indeed, we have $R\left(r, A_{\alpha-1}\right) B x \rightarrow 0$ as $r \rightarrow \infty$ in $X_{\alpha}^{A}$ uniformly for $x$ in $X_{\alpha}^{A}$ with $\|x\|_{\alpha}^{A} \leq 1$.

(B) $\|B\|_{\mathcal{L}\left(X_{\alpha}^{A}, X_{\alpha-1}^{A}\right)} \leq\left(2 c_{0}(1+K)\right)^{-1}$. Here we can take $\bar{\omega}=0$. (Use estimate (2.5).)

(C) $B: X_{\beta}^{A} \longrightarrow X_{\alpha-1}^{A}$ is bounded for some $0 \leq \beta<\alpha$. Indeed, (2.8) implies that

$$
\left\|B R\left(\bar{\omega}, A_{\alpha-1}\right) x\right\|_{\alpha-1}^{A} \leq\|B\|_{\mathcal{L}\left(X_{\beta}^{A}, X_{\alpha-1}^{A}\right)} \bar{c}(1+\bar{\omega})^{\beta-\alpha}\|x\|_{\alpha-1}^{A} \leq q\|x\|_{\alpha-1}^{A}
$$

for $x \in X_{\alpha-1}^{A}$ and a sufficiently large $\bar{\omega} \geq 0$ depending on $\alpha-\beta,\|B\|_{\mathcal{L}\left(X_{\beta}^{A}, X_{\alpha-1}^{A}\right)}, K, \phi$.

(D) $B: X_{\alpha}^{A} \longrightarrow X_{\beta-1}^{A}$ is bounded for some $\alpha<\beta \leq 1$. Indeed, from inequality (2.8) (after interchanging the roles of $\alpha$ and $\beta$ ) we deduce that

$$
\left\|R\left(\bar{\omega}, A_{-1}\right) B x\right\|_{\alpha}^{A} \leq \bar{c}(1+\bar{\omega})^{\alpha-\beta}\|B\|_{\mathcal{L}\left(X_{\alpha}^{A}, X_{\beta-1}^{A}\right)}\|x\|_{\alpha}^{A} \leq q\|x\|_{\alpha}^{A}
$$

for $x \in X_{\alpha}^{A}$ and a sufficiently large $\bar{\omega}$ depending on $\beta-\alpha,\|B\|_{\mathcal{L}\left(X_{\alpha}^{A}, X_{\beta-1}^{A}\right)}, K, \phi$.

For operators $A$ and $B$ satisfying (H1), we denote by $C$ the part of $A_{\alpha-1}+B$ in $X$ defined on the domain $D(C)=\left\{x \in X_{\alpha}^{A}:\left(A_{\alpha-1}+B\right) x \in X\right\}$. We show the sectoriality of $C$ in the following proposition. This result is essentially known (cf. [10] and [12]), but we 
give the proof since we have to determine the precise constants in the framework of our hypothesis (H1).

Proposition 3.2. If (H1) holds, then $C$ is sectorial with the constants $\bar{\omega}, \phi$, and some $\bar{K}$ only depending on $K, \phi$, and $\|B\|_{\mathcal{L}\left(X_{\alpha}^{A}, X_{\alpha-1}^{A}\right)}$. For $\lambda \in \Sigma_{\bar{\omega}, \phi}$, we further have

$$
\begin{aligned}
R(\lambda, C) & =R(\lambda, A)+R\left(\lambda, A_{-1}\right) B\left[I-R\left(\lambda, A_{-1}\right) B\right]^{-1} R(\lambda, A) \\
& =R(\lambda, A)+R\left(\lambda, A_{-1}\right) B R(\lambda, C) . \\
R(\lambda, C) & =R(\lambda, A)+R\left(\lambda, A_{-1}\right)\left[I-B R\left(\lambda, A_{-1}\right)\right]^{-1} B R(\lambda, A) \\
& =R(\lambda, A)+\left(\lambda-A_{\alpha-1}-B\right)^{-1} B R(\lambda, A),
\end{aligned}
$$

Proof. We assume that (H1)(a) holds. The arguments for the case (H1)(b) are analogous (based on (3.4) instead of (3.2)) so that we do not treat this case. Let $\lambda \in \Sigma_{\bar{\omega}, \phi}$. The resolvent equation, (H1)(a), and (2.4) yield

$$
\begin{aligned}
\left\|R\left(\lambda, A_{-1}\right) B\right\|_{\mathcal{L}\left(X_{\alpha}^{A}\right)} & \leq\left\|R\left(\bar{\omega}, A_{-1}\right) B\right\|_{\mathcal{L}\left(X_{\alpha}^{A}\right)}+|\bar{\omega}-\lambda|\left\|R(\lambda, A) R\left(\bar{\omega}, A_{-1}\right) B\right\|_{\mathcal{L}\left(X_{\alpha}^{A}\right)} \\
& \leq q+K q \frac{|\bar{\omega}-\lambda|}{1+|\lambda|} \leq q+\frac{q K}{\sin (\pi-\phi)} \leq \frac{1}{2} .
\end{aligned}
$$

Hence, the inverse $\left(I-R\left(\lambda, A_{-1}\right) B\right)^{-1}$ exists in $\mathcal{L}\left(X_{\alpha}^{A}\right)$ and its norm is bounded by 2 for $\lambda \in \Sigma_{\bar{\omega}, \phi}$. We also have $\left(I-B R\left(\lambda, A_{-1}\right)\right)^{-1} \in \mathcal{L}\left(X_{\alpha-1}^{A}\right)$ with norm less than $1+2(1+$ $K)\|B\|_{\mathcal{L}\left(X_{\alpha}^{A}, X_{\alpha-1}^{A}\right)}$ because of (2.5) and

$$
\left(I-B R\left(\lambda, A_{-1}\right)\right)^{-1}=I+B\left(I-R\left(\lambda, A_{-1}\right) B\right)^{-1} R\left(\lambda, A_{-1}\right) .
$$

We further define

$$
\begin{aligned}
R_{\lambda} & :=R\left(\lambda, A_{-1}\right)+R\left(\lambda, A_{-1}\right) B\left[I-R\left(\lambda, A_{-1}\right) B\right]^{-1} R\left(\lambda, A_{-1}\right) \\
& =R\left(\lambda, A_{-1}\right)+R\left(\lambda, A_{-1}\right)\left[I-B R\left(\lambda, A_{-1}\right)\right]^{-1} B R\left(\lambda, A_{-1}\right)
\end{aligned}
$$

for $\lambda \in \Sigma_{\bar{\omega}, \phi}$, where the second equality follows from (3.7). Using (3.8), (2.8), (3.6), and (2.7), we estimate

$$
\left\|R_{\lambda}\right\|_{\mathcal{L}(X)} \leq \frac{K+2 \bar{c}^{2}\|B\|_{\mathcal{L}\left(X_{\alpha}^{A}, X_{\alpha-1}^{A}\right)}}{1+|\lambda|} \leq \frac{\bar{K}}{1+|\lambda-\bar{\omega}|}
$$

setting $\bar{K}=\left(K+2 \bar{c}^{2}\|B\|_{\mathcal{L}\left(X_{\alpha}^{A}, X_{\alpha-1}^{A}\right)}\right) \frac{1}{\sin (\pi-\phi)}$. Formulas (3.8), (2.5), and (3.6) also yield

$$
\left\|R_{\lambda}\right\|_{\mathcal{L}\left(X_{\alpha-1}^{A}, X_{\alpha}^{A}\right)} \leq 1+K+\frac{2}{2}(1+K)=2(1+K) .
$$

For $x \in X_{\alpha-1}^{A}$ and $\lambda \in \Sigma_{\bar{\omega}, \phi}$, we deduce from (3.8) that

$$
\begin{aligned}
\left(\lambda-A_{\alpha-1}-B\right) R_{\lambda} x= & x-B R\left(\lambda, A_{-1}\right) x+B\left[I-R\left(\lambda, A_{-1}\right) B\right]^{-1} R\left(\lambda, A_{-1}\right) x \\
& \quad-B R\left(\lambda, A_{-1}\right) B\left[I-R\left(\lambda, A_{-1}\right) B\right]^{-1} R\left(\lambda, A_{-1}\right) x \\
=x & ,
\end{aligned}
$$

and for $x \in X_{\alpha}^{A}$ that

$$
\begin{aligned}
R_{\lambda}\left(\lambda-A_{\alpha-1}-B\right) x= & x-R\left(\lambda, A_{-1}\right) B x+R\left(\lambda, A_{-1}\right) B\left[I-R\left(\lambda, A_{-1}\right) B\right]^{-1} x \\
& \quad-R\left(\lambda, A_{-1}\right) B\left[I-R\left(\lambda, A_{-1}\right) B\right]^{-1} R\left(\lambda, A_{-1}\right) B x \\
= & x .
\end{aligned}
$$


As a result, $R_{\lambda}$ is the inverse of $\lambda-A_{\alpha-1}-B \in \mathcal{L}\left(X_{\alpha}^{A}, X_{\alpha-1}^{A}\right)$; i.e., $\lambda \in \rho(C)$ and $R(\lambda, C)$ is the restriction to $X$ of $R_{\lambda}$. In view of (3.10), $C$ is sectorial with the constants $\phi, \bar{\omega}$, and $\bar{K}$. Moreover, the identities (3.2) and (3.4) are consequences of (3.8) and (3.9). Equations (3.3), resp. (3.5), easily follow from (3.2), resp. from (3.4).

The above formula (3.2) leads to the following embeddings of the inter/extrapolation spaces for $A$ and $C$.

Lemma 3.3. Let (H1) hold. Then the following assertions are true.

(i) We have $X_{\alpha}^{A} \hookrightarrow X_{\alpha}^{C}$ and $X_{\alpha-1}^{A} \hookrightarrow X_{\alpha-1}^{C}$, where the norms of the embeddings are bounded by a constant only depending on $K, \phi, \bar{\omega}$, and $\|B\|_{\mathcal{L}\left(X_{\alpha}^{A}, X_{\alpha-1}^{A}\right)}$.

(ii) We have $X_{\alpha+\epsilon}^{C} \hookrightarrow X_{\alpha}^{A}$ for $0<\epsilon \leq 1-\alpha$, where the norm of the embedding is bounded by a constant only depending on $K, \phi, \bar{\omega}$, and $\epsilon$.

Proof. As in Lemma 3.2, we only consider the assumption (H1)(a) since the case (H1)(b) can be proved similarly.

(i) We treat the first embedding. For $\lambda>0$ and $x \in X_{\alpha}^{A}$, equality (3.2) yields

$$
\begin{aligned}
\lambda^{\alpha}(C-\bar{\omega}) R(\lambda, C-\bar{\omega}) x= & \lambda^{1+\alpha} R(\lambda, C-\bar{\omega})-\lambda^{\alpha} x \\
= & {\left[\lambda^{1+\alpha} R(\lambda, A-\bar{\omega}) x-\lambda^{\alpha} x\right] } \\
& +\lambda^{\alpha} R\left(\lambda, A_{-1}-\bar{\omega}\right) B\left[I-R\left(\lambda, A_{-1}-\bar{\omega}\right) B\right]^{-1} \lambda R\left(\lambda, A_{\alpha}-\bar{\omega}\right) x \\
= & : S_{1}^{\lambda} x+S_{2}^{\lambda} x .
\end{aligned}
$$

The sectoriality of $A$ combined with the results in Section 2.2.1 of [15] implies that

$$
\begin{aligned}
\left\|S_{1}^{\lambda} x\right\| & =\left\|(A-\bar{\omega}) A^{-1}(\lambda-A) R(\lambda, A-\bar{\omega}) \lambda^{\alpha} A R(\lambda, A) x\right\| \\
& \leq(1+\bar{\omega} K)(1+2 K)\left\|\lambda^{\alpha} A R(\lambda, A) x\right\| \quad \longrightarrow 0
\end{aligned}
$$

as $\lambda \rightarrow \infty$. Using (2.8) and (3.6), we further estimate

$$
\left\|S_{2}^{\lambda} x\right\| \leq \frac{2 \bar{c} \lambda^{\alpha}}{(\lambda+\bar{\omega})^{\alpha}}\|B\|_{\mathcal{L}\left(X_{\alpha}^{A}, X_{\alpha-1}^{A}\right)}\left\|\lambda R\left(\lambda, A_{\alpha}-\bar{\omega}\right) x\right\|_{\alpha}^{A} .
$$

Since $A_{\alpha}$ is densely defined in $X_{\alpha}^{A}$, the term $S_{2}^{\lambda} x$ converges to 0 as $\lambda \rightarrow \infty$. As a result, $x$ belongs to $X_{\alpha}^{C}$ by $[15, \S 2.2 .1]$. Moreover, the estimates (3.12), (3.13), and (2.4) yield

$$
\left\|S_{1}^{\lambda} x+S_{2}^{\lambda} x\right\| \leq(1+\bar{\omega} K)(1+2 K)\|x\|_{\alpha}^{A}+2 \bar{c} K\|B\|_{\mathcal{L}\left(X_{\alpha}^{A}, X_{\alpha-1}^{A}\right)}\|x\|_{\alpha}^{A}
$$

for $\lambda>0$, so that $X_{\alpha}^{A} \hookrightarrow X_{\alpha}^{C}$ and

$$
\|x\|_{\alpha}^{C} \leq c\left(K,\|B\|_{\mathcal{L}\left(X_{\alpha}^{A}, X_{\alpha-1}^{A}\right)}, \bar{\omega}\right)\|x\|_{\alpha}^{A} .
$$

For the second embedding, we identify $X_{\alpha-1}^{A}$ with $\left(X_{\alpha}^{A},\|\cdot\|_{\alpha-1}^{A}\right)^{\sim}$ via the isomorphism

$$
J_{0}: X_{\alpha-1}^{A} \rightarrow\left(X_{\alpha}^{A},\|\cdot\|_{\alpha-1}^{A}\right)^{\sim} ; \quad J_{0} x=\left(x_{n}\right)+\mathcal{N}_{\alpha-1}^{A},
$$

where $x_{n} \in X_{\alpha}^{A}$ with $x_{n} \rightarrow x$ in $X_{\alpha-1}^{A}$ as $n \rightarrow \infty$ and $\mathcal{N}_{\alpha-1}^{A}=\left\{\left(y_{n}\right) \subset X_{\alpha}^{A}: y_{n} \rightarrow\right.$ 0 in $X_{\alpha-1}^{A}$ as $\left.n \rightarrow \infty\right\}$. From (3.11) we know that $R(\lambda, C)$ has the continuous injective extension $\left(\lambda-A_{\alpha-1}-B\right)^{-1}: X_{\alpha-1}^{A} \rightarrow X_{\alpha}^{A}$ for $\lambda \geq \bar{\omega}$. Let $x \in X_{\alpha-1}^{A}$ and $\left(x_{n}\right) \subset X_{\alpha}^{A}$ be a sequence with limit $x$ in $X_{\alpha-1}^{A}$. Using (3.14) and (3.11), we estimate

$$
\begin{aligned}
\left\|x_{n}-x_{m}\right\|_{\alpha-1}^{C} & =\left\|R(\bar{\omega}, C)\left(x_{n}-x_{m}\right)\right\|_{\alpha}^{C} \leq c\left\|R(\bar{\omega}, C)\left(x_{n}-x_{m}\right)\right\|_{\alpha}^{A} \\
& \leq c\left\|x_{n}-x_{m}\right\|_{\alpha-1}^{A} .
\end{aligned}
$$


So we can define the linear map

$$
J: X_{\alpha-1}^{A} \longrightarrow X_{\alpha-1}^{C} \cong\left(X_{\alpha-1}^{C},\|\cdot\|_{\alpha-1}^{C}\right)^{\sim} ; \quad J x=\left(x_{n}\right)+\mathcal{N}_{\alpha-1}^{C}
$$

(Observe that we can identify $J X_{\alpha}^{A}$ with $X_{\alpha}^{A}$.) As in (3.15), it follows

$$
\|J x\|_{\alpha-1}^{C}=\lim _{n \rightarrow \infty}\left\|x_{n}\right\|_{\alpha-1}^{C} \leq c\left(K,\|B\|_{\mathcal{L}\left(X_{\alpha}, X_{\alpha-1}\right)}, \bar{\omega}\right)\|x\|_{\alpha-1}^{A} .
$$

If $J x=0$, then there are $x_{n} \in X_{\alpha}^{A}$ with $x_{n} \rightarrow 0$ in $X_{\alpha-1}^{C}$ and $x_{n} \rightarrow x$ in $X_{\alpha-1}^{A}$. Hence, $R(\bar{\omega}, C) x_{n} \rightarrow 0$ in $X_{\alpha}^{C}$ and $R(\bar{\omega}, C) x_{n} \rightarrow\left(\bar{\omega}-A_{\alpha-1}-B\right)^{-1} x$ in $X_{\alpha}^{A} \hookrightarrow X_{\alpha}^{C}$. Therefore $\left(\bar{\omega}-A_{\alpha-1}-B\right)^{-1} x=0$, and then $x=0$. So we have shown that $X_{\alpha-1}^{A} \hookrightarrow X_{\alpha-1}^{C}$.

(ii) For $\lambda \in \Sigma_{\bar{\omega}, \phi}$, the identity (3.2) and the inequalities (2.7) and (3.6) imply

$$
\|R(\lambda, C)\|_{\mathcal{L}\left(X, X_{\alpha}^{A}\right)} \leq \frac{\bar{c}}{(1+|\lambda|)^{1-\alpha}}+\frac{2 \bar{c}}{2(1+|\lambda|)^{1-\alpha}}=\frac{2 \bar{c}}{(1+|\lambda|)^{1-\alpha}} .
$$

Taking $\alpha<\theta<\alpha+\epsilon \leq 1$ and $x \in X$, we can thus estimate

$$
\left\|(\bar{\omega}-C)^{-\theta} x\right\|_{\alpha} \leq \frac{\bar{c}}{\pi} \int_{\Gamma}|\lambda-\bar{\omega}|^{-\theta}(1+|\lambda|)^{\alpha-1}\|x\||d \lambda| \leq c(K, \bar{\omega}, \phi, \theta)\|x\|
$$

for a suitable path $\Gamma$ in $\mathbb{C}$. This gives the assertion since $X_{\alpha+\epsilon}^{C} \hookrightarrow D\left((\bar{\omega}-C)^{\theta}\right)$.

In the following result we even obtain equality of the inter/extrapolation spaces of $A$ and $C$ under stronger assumptions on $B$, cf. Remark 3.1. For exponents strictly between $\alpha$ and $\alpha-1$, such identities were also shown in [10, Thm.5.3] in a more general setting using different methods.

Proposition 3.4. Let $A$ be sectorial with constants $K \geq 0, \phi \in(\pi / 2, \pi)$, and $\omega=0$. Assume that either $B \in \mathcal{L}\left(X_{\beta}^{A}, X_{\alpha-1}^{A}\right)$ for some $\beta \in[0, \alpha)$, or $B \in \mathcal{L}\left(X_{\alpha}^{A}, X_{\beta-1}^{A}\right)$ for some $\beta \in(\alpha, 1]$, or $\|B\|_{\mathcal{L}\left(X_{\alpha}^{A}, X_{\alpha-1}^{A}\right)} \leq q_{0}:=\left[4(1+K) c_{0}\right]^{-1}$. Then $X_{\alpha}^{A}=X_{\alpha}^{C}$ and $X_{\alpha-1}^{A} \cong X_{\alpha-1}^{C}$, where the respective norms are equivalent with constants $c\left(K, \bar{\omega}, \phi, \alpha, \beta,\|B\|_{\mathcal{L}\left(X_{\beta}^{A}, X_{\alpha-1}^{A}\right)}\right)$, $c\left(K, \bar{\omega}, \phi, \alpha, \beta,\|B\|_{\mathcal{L}\left(X_{\alpha}^{A}, X_{\beta-1}^{A}\right)}\right)$ and $c(K, \phi, \bar{\omega})$, respectively.

Proof. Step 1. We first show the equivalence of $\|\cdot\|_{\alpha}^{A}$ and $\|\cdot\|_{\alpha}^{C}$ on $D(C)$, respectively, of $\|\cdot\|_{\alpha-1}^{A}$ and $\|\cdot\|_{\alpha-1}^{C}$ on $X$, in each of the three cases.

(i) Assume that $\|B\|_{\mathcal{L}\left(X_{\alpha}^{A}, X_{\alpha-1}^{A}\right)} \leq q_{0}$. Then (H1)(a) holds with $\bar{\omega}=0$ by Remark 3.1, and Lemma 3.3 yields $X_{\alpha}^{A} \hookrightarrow X_{\alpha}^{C}$ and $X_{\alpha-1}^{A} \hookrightarrow X_{\alpha-1}^{C}$. For the converse implications, take $\lambda>0$ and $x \in D(C) \hookrightarrow X_{\alpha}^{A}$ (see Lemma 3.3). Equality (3.2) implies that

$$
\begin{aligned}
\lambda^{\alpha} A R(\lambda, A) x-\lambda^{\alpha} C R(\lambda, C) x & =\lambda^{1+\alpha} R(\lambda, A) x-\lambda^{1+\alpha} R(\lambda, C) x \\
& =-\lambda^{\alpha} R\left(\lambda, A_{-1}\right) B\left(I-R\left(\lambda, A_{-1}\right) B\right)^{-1} \lambda R(\lambda, A) x .
\end{aligned}
$$

Using (2.4), we estimate

$$
\begin{aligned}
& \|x\|_{\alpha}^{A} \leq\|x\|_{\alpha}^{C}+\|B\|_{\mathcal{L}\left(X_{\alpha}^{A}, X_{\alpha-1}^{A}\right)} 2 K\|x\|_{\alpha}^{A} \leq\|x\|_{\alpha}^{C}+\frac{1}{2}\|x\|_{\alpha}^{A}, \\
& \|x\|_{\alpha}^{A} \leq 2\|x\|_{\alpha}^{C} .
\end{aligned}
$$

Similarly, for $x \in X \hookrightarrow X_{\alpha-1}^{A}$ we obtain

$$
\begin{aligned}
& \|x\|_{\alpha-1}^{A} \leq\|x\|_{\alpha-1}^{C}+\|B\|_{\mathcal{L}\left(X_{\alpha}^{A}, X_{\alpha-1}^{A}\right)} 2(1+K)\|x\|_{\alpha-1}^{A} \leq\|x\|_{\alpha-1}^{C}+\frac{1}{2}\|x\|_{\alpha-1}^{A}, \\
& \|x\|_{\alpha-1}^{A} \leq 2\|x\|_{\alpha-1}^{C} .
\end{aligned}
$$


(ii) Let $B \in \mathcal{L}\left(X_{\beta}^{A}, X_{\alpha-1}^{A}\right)$ for $0 \leq \beta<\alpha$. Then (H1)(b) holds for some $\bar{\omega} \geq 0$ by Remark 3.1, and hence $X_{\alpha}^{A} \hookrightarrow X_{\alpha}^{C}$ and $X_{\alpha-1}^{A} \hookrightarrow X_{\alpha-1}^{C}$ by Lemma 3.3. Let $\lambda \geq 0$. From (3.5) we deduce that

$$
\begin{aligned}
& \lambda^{\alpha}(A-\bar{\omega}) R(\lambda, A-\bar{\omega}) \\
& \quad=\lambda^{\alpha}(C-\bar{\omega}) R(\lambda, C-\bar{\omega})-\lambda^{\alpha}\left(\lambda+\bar{\omega}-A_{\alpha-1}-B\right)^{-1} B \lambda R(\lambda, A-\bar{\omega}) .
\end{aligned}
$$

For $x \in D(C) \subset X_{\alpha}^{A}$ (see Lemma 3.3), this identity yields

$$
\begin{aligned}
\|x\|_{\alpha}^{A} & \leq c\|x\|_{\alpha}^{C}+c \sup _{\lambda>0}\|B \lambda R(\lambda, A-\bar{\omega}) x\|_{\alpha-1}^{C} \\
& \leq c\|x\|_{\alpha}^{C}+c\|B\|_{\mathcal{L}\left(X_{\beta}^{A}, X_{\alpha-1}^{A}\right)} \sup _{\lambda>0}\|\lambda R(\lambda, A-\bar{\omega}) x\|_{\beta}^{A} \\
& \leq c\|x\|_{\alpha}^{C}+c\|x\|_{\beta}^{A},
\end{aligned}
$$

where $c=c\left(K, \phi, \bar{\omega},\|B\|_{\mathcal{L}\left(X_{\beta}^{A}, X_{\alpha-1}^{A}\right)}\right)$ and we have used Lemma 3.3 and (2.4). We further obtain $X_{\frac{\beta}{\alpha}}^{C} \hookrightarrow X_{\beta}^{A}$ by interpolating $X_{1}^{C} \hookrightarrow X_{\alpha}^{A}$ and $X \hookrightarrow X$. Consequently,

$$
\|x\|_{\alpha}^{A} \leq c\|x\|_{\alpha}^{C}+c\|x\|_{\frac{\beta}{\alpha}}^{C} .
$$

If $\frac{\beta}{\alpha} \leq \alpha$, we arrive at

$$
\|x\|_{\alpha}^{A} \leq c\|x\|_{\alpha}^{C}
$$

Otherwise, we deduce that

$$
\|x\|_{\alpha}^{A} \leq c\|x\|_{\frac{\beta}{\alpha}}^{C} .
$$

Hence, $X_{\frac{\beta^{2}}{\alpha^{2}}}^{C} \hookrightarrow X_{\beta}^{A}$ by interpolation. So (3.17) yields

$$
\|x\|_{\alpha}^{A} \leq c\|x\|_{\alpha}^{C}+c\|x\|_{\frac{\beta^{2}}{\alpha^{2}}}^{C} .
$$

We now iterate until $\frac{\beta^{n}}{\alpha^{n}} \leq \alpha$ arriving at (3.18) with $c=c\left(K, \bar{\omega}, \phi, \alpha, \beta,\|B\|_{\mathcal{L}\left(X_{\beta}^{A}, X_{\alpha-1}^{A}\right)}\right)$. As a consequence, $R(\mu, C)$ has a uniformly bounded extesnion from $X_{\alpha-1}^{C}$ to $X_{\alpha}^{A}$ for $\mu \geq \bar{\omega}$. By means of this fact and (3.3), we estimate

$$
\begin{aligned}
\|x\|_{\alpha-1}^{A} & \leq c \sup _{\lambda>0}\left\|\lambda^{\alpha} R(\lambda, A-\bar{\omega}) x\right\| \\
& \leq c\|x\|_{\alpha-1}^{C}+c \sup _{\lambda>0}\left\|\lambda^{\alpha} R(\lambda, A-\bar{\omega}) B R(\lambda, C-\bar{\omega}) x\right\| \\
& \leq c\|x\|_{\alpha-1}^{C}+c\|B\|_{\mathcal{L}\left(X_{\beta}^{A}, X_{\alpha-1}^{A}\right)} \sup _{\lambda>0}\|R(\lambda, C-\bar{\omega}) x\|_{\alpha}^{A} \\
& \leq c\|x\|_{\alpha-1}^{C},
\end{aligned}
$$

where $c=c\left(K, \bar{\omega}, \phi, \alpha, \beta,\|B\|_{\mathcal{L}\left(X_{\beta}^{A}, X_{\alpha-1}^{A}\right)}\right)$.

(iii) Let $B: X_{\alpha}^{A} \longrightarrow X_{\beta-1}^{A}$ be bounded for some $\alpha<\beta \leq 1$. Hence (H1)(a) holds by Remark 3.1 so that $X_{\alpha}^{A} \hookrightarrow X_{\alpha}^{C}$ and $X_{\alpha-1}^{A} \hookrightarrow X_{\alpha-1}^{C}$ by Lemma 3.3. For $x \in X_{\alpha}^{A}$, formula (3.16) and Lemma 3.3(ii) yield

$$
\begin{aligned}
\|x\|_{\alpha}^{A} & \leq c \sup _{\lambda>0}\left\{\left\|\lambda^{\alpha}(C-\bar{\omega}) R(\lambda, C-\bar{\omega}) x\right\|+c\left\|\lambda^{\beta} R\left(\lambda, A_{-1}-\bar{\omega}\right) B \lambda^{1+\alpha-\beta} R(\lambda, C-\bar{\omega}) x\right\|\right\} \\
& \leq c\|x\|_{\alpha}^{C}+c\|B\|_{\mathcal{L}\left(X_{\alpha}^{A}, X_{\beta-1}^{A}\right)} \sup _{\lambda>0}\left\|\lambda^{1+\alpha-\beta} R(\lambda, C-\bar{\omega}) x\right\|_{\alpha}^{A}
\end{aligned}
$$




$$
\leq c\|x\|_{\alpha}^{C}+c \sup _{\lambda>0}\|(1+\lambda) R(\lambda, C-\bar{\omega}) x\|_{\beta}^{C} \leq c\|x\|_{\alpha}^{C},
$$

where $c=c\left(K, \bar{\omega}, \phi, \alpha, \beta,\|B\|_{\mathcal{L}\left(X_{\alpha}^{A}, X_{\beta-1}^{A}\right)}\right)$. The inequality (3.19) can be shown similarly.

Step 2. Observe that the embeddings $D(C) \hookrightarrow X_{\alpha}^{A} \hookrightarrow X_{\alpha}^{C}$, respectively $X \hookrightarrow X_{\alpha-1}^{A} \hookrightarrow$ $X_{\alpha-1}^{C}$, are dense in $\|\cdot\|_{\alpha}^{C}$, respectively, in $\|\cdot\|_{\alpha-1}^{C}$. Thus we also obtain $X_{\alpha}^{A}=X_{\alpha}^{C}$ and $X_{\alpha-1}^{A} \cong X_{\alpha-1}^{C}$ (where the isomorphism is given by the embedding $J$ constructed in the proof of Lemma 3.3.)

We turn our attention to the nonautonomous situation and state our main hypothesis.

(H2) There are operators $A(t)$ satisfying (2.1) and (2.2) for $t \in \mathbb{R}$. For every $t \in \mathbb{R}$ and some $\alpha \in(1-\mu, 1)$, there is a Banach space $X_{\alpha}$ with $X_{\alpha}^{t} \hookrightarrow X_{\alpha} \hookrightarrow X$ and there are operators $B(t) \in \mathcal{L}\left(X_{\alpha}, X_{\alpha-1}^{A(t)}\right)$ such that the norms of the embeddings and of $B(t)$ are bounded by a constant $b,(\mathrm{H} 1)$ holds for $A_{\omega}(t)=A(t)-\omega$ and $B(t)$ with uniform constants, and

$$
\left\|R\left(\tilde{\omega}, A_{\alpha-1}(t)\right) B(t) x-R\left(\tilde{\omega}, A_{\alpha-1}(s)\right) B(s) x\right\|_{\alpha}^{t} \leq \ell|t-s|^{\mu}\|x\|_{\alpha}
$$

for some $\ell>0$ and $\tilde{\omega} \geq \omega$ and each $x \in X_{\alpha}, t, s \in \mathbb{R}$. If $\alpha>\nu$, we assume in addition that norms of $X_{\alpha}$ and $X_{\alpha}^{t}$ are uniformly equivalent for $t \in \mathbb{R}$ and that the map $\mathbb{R} \ni t \longmapsto R(\omega, A(t)) \in \mathcal{L}\left(X, X_{\alpha}\right)$ is uniformly Hölder continuous with exponent $\mu$ and constant $\ell$.

In the standard applications, the spaces $X_{\alpha}^{t}$ are in fact closed subspaces of a space $X_{\alpha}$ and $R(\omega, A(\cdot))$ is Hölder continuous in $\mathcal{L}\left(X, X_{\alpha}\right)$, see e.g. Example 5.9.

Remark 3.5. (i) If (H2) holds for $s$ and $t$ in a closed interval $J \subset \mathbb{R}$, we can extend $A(t)$, $B(t), X_{\alpha}^{t}$, and $X_{\alpha-1}^{t}$ constantly to $t \in \mathbb{R}$. The extensions satisfy again (H2) on $\mathbb{R}$ with the same constants.

(ii) If condition (2.2) holds for some $\omega \in \mathbb{R}$, then it is true for each $\omega^{\prime} \geq \omega$ with the same constants $\mu, \nu, \phi$, and a constant $L^{\prime}=L^{\prime}(L, K, \omega)$. Indeed, formula (2.8) in [18] yields

$$
\begin{aligned}
& \left(A(t)-\omega^{\prime}\right) R\left(\lambda+\omega^{\prime}, A(t)\right)\left[R\left(\omega^{\prime}, A(t)\right)-R\left(\omega^{\prime}, A(s)\right)\right] \\
& =\left(A_{\omega}(t)-\lambda\right) R\left(\lambda+\omega^{\prime}, A(t)\right) A_{\omega}(t) R\left(\lambda, A_{\omega}(t)\right)[R(\omega, A(t))-R(\omega, A(s))] A_{\omega}(s) R\left(\omega^{\prime}, A(s)\right)
\end{aligned}
$$

for $\lambda \in \Sigma_{\phi}$ and $t, s \in \mathbb{R}$. The resolvent equation further shows that the last condition in (H2) still holds (possibly with a different $\ell$ ) if one replaces $\omega$ by a number $\lambda \geq \omega$.

(iii) Let (H2) hold. Then (3.20) is also true for $\lambda \geq \omega$ instead of $\tilde{\omega}$ with the same $\mu$ and $\alpha$, and an $\bar{\ell}$ depending on $\lambda$ and the constants in (H2). This fact follows from (H2) and the equality

$$
\begin{aligned}
& R\left(\lambda, A_{\alpha-1}(t)\right) B(t)-R\left(\lambda, A_{\alpha-1}(s)\right) B(s) \\
&= R\left(\tilde{\omega}, A_{\alpha-1}(t)\right) B(t)-R\left(\tilde{\omega}, A_{\alpha-1}(s)\right) B(s) \\
&+(\tilde{\omega}-\lambda) R(\lambda, A(t))\left[R\left(\tilde{\omega}, A_{\alpha-1}(t)\right) B(t)-R\left(\tilde{\omega}, A_{\alpha-1}(s)\right) B(s)\right] \\
&+(\tilde{\omega}-\lambda)[R(\lambda, A(t))-R(\lambda, A(s))] R\left(\tilde{\omega}, A_{\alpha-1}(s)\right) B(s) .
\end{aligned}
$$

(iv) Let (H2) hold. Then the map $t \longmapsto R\left(\lambda, A_{\alpha-1}(t)\right) B(t) R(\mu, A(t)) \in \mathcal{L}(X)$ is Hölder continuous for $\lambda, \mu \geq \max \{\tilde{\omega}, \bar{\omega}\}$. This fact follows from (H2) and parts (ii) and (iii).

Let (H2) hold. Due to Proposition 3.2 there exists the part $C(t)$ of $A_{\alpha-1}(t)+B(t)$ in $X$ for every $t \in \mathbb{R}$. We next prove that the operators $C(t)$ also satisfy condition (2.2). 
Proposition 3.6. Assume that (H2) holds. Then the operators $C(t), t \in \mathbb{R}$, fulfill (2.1) and (2.2) with the constants $\bar{K}, \phi, \bar{\omega}, \mu, \min \{\nu, \alpha\}$ (instead of $\nu$ ), and some $\bar{L}=\bar{L}(L, K, \phi, \bar{\omega}, b, \ell)$. Therefore $C(\cdot)$ generates an evolution family $U_{B}(\cdot, \cdot)$ satisfying the assertions of Proposition 2.1 with $U(\cdot, \cdot)$ replaced by $U_{B}(\cdot, \cdot)$ and $A(\cdot)$ by $C(\cdot)$.

Proof. Proposition 3.2 shows that (2.1) holds. Concerning (2.2), we only consider the case $\alpha \leq \nu$. The case $\alpha>\nu$ is treated in the same way. Using formula (3.2), we write

$$
\begin{aligned}
& {[R(\bar{\omega}, C(t))-R(\bar{\omega}, C(s))] x=[R(\bar{\omega}, A(t))-R(\bar{\omega}, A(s))] x} \\
& \quad+R\left(\bar{\omega}, A_{-1}(t)\right) B(t)\left[I-R\left(\bar{\omega}, A_{-1}(t)\right) B(t)\right]^{-1}[R(\bar{\omega}, A(t))-R(\bar{\omega}, A(s))] x \\
& \quad+R\left(\bar{\omega}, A_{-1}(t)\right) B(t)\left(I-R\left(\bar{\omega}, A_{-1}(t)\right) B(t)\right)^{-1}\left[R\left(\bar{\omega}, A_{-1}(t)\right) B(t)-R\left(\bar{\omega}, A_{-1}(s)\right) B(s)\right] \\
& \quad \cdot\left(I-R\left(\bar{\omega}, A_{-1}(s)\right) B(s)\right)^{-1} R(\bar{\omega}, A(s)) x \\
& \quad+\left[R\left(\bar{\omega}, A_{-1}(t)\right) B(t)-R\left(\bar{\omega}, A_{-1}(s)\right) B(s)\right]\left(I-R\left(\bar{\omega}, A_{-1}(s)\right) B(s)\right)^{-1} R(\bar{\omega}, A(s)) x
\end{aligned}
$$

for $x \in X$ and $t, s \in \mathbb{R}$. The above equation, Remark 3.5, (3.6) and (H2) yield that $[R(\bar{\omega}, C(t))-R(\bar{\omega}, C(s))] x \in X_{\alpha}^{A(t)}$. Taking also into account Proposition 3.2 and Lemma 3.3, we then deduce

$$
\begin{aligned}
\sup _{\lambda \in \Sigma_{\phi}} \| & \lambda^{\alpha}(C(t)-\bar{\omega}) R(\lambda, C(t)-\bar{\omega})[R(\bar{\omega}, C(t))-R(\bar{\omega}, C(s))] x \| \\
= & \sup _{\lambda \in \Sigma_{\phi}} \|(|\lambda|+\bar{\omega}-C(t)) R(\lambda, C(t)-\bar{\omega})|\lambda|^{\alpha}(C(t)-\bar{\omega}) R(|\lambda|, C(t)-\bar{\omega}) \\
& \cdot[R(\bar{\omega}, C(t))-R(\bar{\omega}, C(s))] x \| \\
\leq & (1+2 \bar{K}) \sup _{\mu>0}\left\|\mu^{\alpha}(C(t)-\bar{\omega}) R(\mu, C(t)-\bar{\omega})[R(\bar{\omega}, C(t))-R(\bar{\omega}, C(s))] x\right\| \\
\leq & c\|[R(\bar{\omega}, C(t))-R(\bar{\omega}, C(s))] x\|_{\alpha}^{A(t)} \leq c|t-s|^{\mu}\|x\| .
\end{aligned}
$$

We now come to the main result of this section relating the evolution family $U_{B}(\cdot, \cdot)$ with $U(\cdot, \cdot)$. In formula $(3.22)$ below we identify $X_{\alpha-1}^{A(t)}$ with subspace of $X_{\alpha-1}^{C(t)}$ be means of the embedding $J$ constructed in Lemma 3.3.

Theorem 3.7. Let $1-\mu<\alpha<1$ and assume that the operators $A(t)$ and $B(t), t \in \mathbb{R}$, satisfy (H2). For $x \in X_{\beta-1}^{A(s)}, \alpha<\beta \leq 1$ and $t \geq s$, it holds

$$
\begin{aligned}
& U_{B, \alpha-1}(t, s) x=U_{\alpha-1}(t, s) x+\int_{s}^{t} U_{\alpha-1}(t, \tau) B(\tau) U_{B, \alpha-1}(\tau, s) x d \tau, \\
& U_{B, \alpha-1}(t, s) x=U_{\alpha-1}(t, s) x+\int_{s}^{t} U_{B, \alpha-1}(t, \tau) B(\tau) U_{\alpha-1}(\tau, s) x d \tau .
\end{aligned}
$$

Proof. Take $x \in D(C(s)) \hookrightarrow X_{\alpha}^{A(s)}$ and $\tau \in(s, t)$. Then $U_{B}(\tau, s) x \in X_{\alpha}^{A(\tau)}$ by Proposition 3.6 and Lemma 3.3, so that Proposition 2.1 yields

$$
\begin{aligned}
\frac{\partial^{+}}{\partial \tau} U(t, \tau) U_{B}(\tau, s) x & =U_{\alpha-1}(t, \tau)\left(A_{\alpha-1}(\tau)+B(\tau)\right) U_{B}(\tau, s) x-U_{\alpha-1}(t, \tau) A_{\alpha-1}(\tau) U_{B}(\tau, s) x \\
& =U_{\alpha-1}(t, \tau) B(\tau) U_{B}(\tau, s) x=: f(\tau),
\end{aligned}
$$

where $\|f(\tau)\| \leq c|t-\tau|^{\alpha-1}\|x\|_{1}^{C(s)}$. Due to Remark 3.5(iv), the function

$$
f_{n}(\tau)=U(t, \tau) n R\left(n, A_{\alpha-1}(\tau)\right) B(\tau) n R(n, A(\tau)) U_{B}(\tau, s) x
$$

is continuous from $[s, t]$ to $X$ for large $n \in \mathbb{N}$. Moreover, $f_{n}(\tau) \rightarrow f(\tau)$ in $X$ as $n \rightarrow \infty$ for $\tau \in[s, t)$ locally uniformly because of (2.13) and (2.8), so that $f \in C([s, t), X) \cap$ 
$L^{1}([s, t), X)$. As a consequence,

$$
U(t, t-\epsilon) U_{B}(t-\epsilon, s) x-U(t, s) x=\int_{s}^{t-\epsilon} \frac{\partial^{+}}{\partial \tau} U(t, \tau) U_{B}(\tau, s) x d \tau=\int_{s}^{t-\epsilon} f(\tau) d \tau
$$

for $0<\epsilon<t-s$. Letting $\epsilon \rightarrow 0$, equation (3.21) follows for $x \in D(C(s)$ ). Using Lemma 3.3, we then obtain (3.21) for $x \in X_{\alpha}^{A(s)}$ by approximation in $X_{\alpha}^{C(s)} \hookrightarrow X$. In a second step we approximate a given $x \in X_{\beta-1}^{A(s)}$ with $\alpha<\beta<1$ in $X_{\beta-1}^{A(s)}$ by $x_{n} \in X_{\alpha}^{A(s)}$ in order to derive the first assertion, again employing Lemma 3.3.

For the second formula, take $\tau \in(s, t)$ and $x \in X_{\alpha}^{A(s)}$. Lemma 3.3 and Propositions 2.1 and 3.6 imply that

$$
\begin{aligned}
\frac{\partial^{+}}{\partial \tau} U_{B}(t, \tau) U(\tau, s) x & =-U_{B, \alpha-1}(t, \tau)\left(A_{\alpha-1}(\tau)+B(\tau)\right) U(\tau, s) x+U_{B}(t, s) A(\tau) U(\tau, s) x \\
& =-U_{B, \alpha-1}(t, \tau) B(\tau) U(\tau, s) x=: g(\tau),
\end{aligned}
$$

where $\|g(\tau)\| \leq c|t-\tau|^{\alpha-1}$ for $\tau \in[s, t)$. Arguing as above, we then derive (3.22) first for $x \in X_{\alpha}^{A(s)}$ and then for $X_{\beta-1}^{A(s)}$ by approximation.

As in [16], we can now study the inhomogeneous evolution equation

$$
\begin{aligned}
& u^{\prime}(t)=C(t) u(t)+f(t), \quad t \in J, \\
& u\left(t_{0}\right)=x,
\end{aligned}
$$

for a closed interval $J, t_{0} \in J, x_{0} \in X$, and $f \in E_{\alpha-1}^{C(\cdot)}$. A function $u \in C(J, X)$ is a mild solution of (3.23) on $J$ if

$$
u(t)=U_{B}(t, s) u(s)+\int_{s}^{t} U_{B, \alpha-1}(t, \tau) f(\tau) d \tau
$$

for all $t, s \in J$ with $t \geq s$. It is a mild solution of the Cauchy problem (3.23)-(3.24) if in addition $u\left(t_{0}\right)=x_{0}$. We recall that a mild solution of (3.23) satisfies (3.23) pointwise in $X_{\beta-1}^{C(t)}$ for $\beta<\alpha$ and $t \in J \backslash \inf J$ due to Proposition 2.6 of [16]. On $J=\mathbb{R}$, we define the closed operator

$$
G_{B, \alpha-1}: D\left(G_{B, \alpha-1}\right) \subseteq E \longrightarrow E_{\alpha-1}^{C(\cdot)} ; \quad G_{B, \alpha-1} u=f,
$$

where $u$ is the mild solution of (3.23) on $\mathbb{R}$, cf. [16, Rem.2.5]. (Here $E=E(\mathbb{R})$ and $\left.E_{\alpha-1}^{C(\cdot)}=E_{\alpha-1}^{C(\cdot)}(\mathbb{R}).\right)$ We further have the analogous operator $G_{\alpha-1}$ on $E_{\alpha-1}^{A(\cdot)}$ given by $U(\cdot, \cdot)$. The following result relates these two operators.

Proposition 3.8. Assume that (H2) holds. Then the following assertions are true.

(i) $E_{\beta}^{A(\cdot)} \hookrightarrow E_{\beta}^{C(\cdot)}$ for $\beta \in[0, \alpha]$ and $E_{\beta-1}^{A(\cdot)} \hookrightarrow E_{\beta-1}^{C(\cdot)}$ for $\beta \in[\alpha, 1]$.

(ii) Assume that the operators $B(t)$ satisfy one of the conditions in Proposition 3.4 with norms uniformly bounded in $t \in \mathbb{R}$. Then $E_{\alpha}^{A(\cdot)}=E_{\alpha}^{C(\cdot)}$ and $E_{\alpha-1}^{A(\cdot)} \cong E_{\alpha-1}^{C(\cdot)}$.

(iii) Let $\gamma \in(\alpha, 1]$ and $f \in E_{\gamma-1}^{A(\cdot)}$. Assume that $B(t), t \in \mathbb{R}$, has a uniformly bounded restriction $B(t): X_{\gamma}^{t} \longrightarrow X_{\gamma-1}^{t}$. If $G_{\alpha-1} u=f$ for some $u \in D\left(G_{\alpha-1}\right)$, then $u \in D\left(G_{B, \alpha-1}\right)$ and $G_{B, \alpha-1} u=G_{\alpha-1} u-B(\cdot) u$. If $G_{B, \alpha-1} v=f$ for some $v \in D\left(G_{B, \alpha-1}\right)$, then $v \in D\left(G_{\alpha-1}\right)$ and $G_{\alpha-1} v=G_{B, \alpha-1} v+B(\cdot) v$.

(iv) Assume that $B(t), t \in \mathbb{R}$, has a uniformly bounded extension $B(t): X_{\beta}^{t} \longrightarrow X_{\gamma-1}^{t}$ for some $\beta \in[0, \alpha)$ and $\gamma \in(\alpha, 1]$. Then $D\left(G_{\alpha-1}\right)=D\left(G_{B, \alpha-1}\right) \subset D(B(\cdot))=\{u \in E$ : $\left.u(t) \in X_{\alpha}^{t}(\forall t \in \mathbb{R}), B(\cdot) u \in E_{\alpha-1}\right\}$ and $G_{B, \alpha-1}=G_{\alpha-1}-B(\cdot)$. 
Proof. In assertion (i), it suffices to consider the case $\beta=\alpha$ since the general case then follows by interpolation. The first embedding can be proved as the corresponding one in Lemma 3.3(i). Let $J(t): X_{\alpha-1}^{A(t)} \rightarrow X_{\alpha-1}^{C(t)}, t \in \mathbb{R}$, be the embeddings constructed in the proof of Lemma 3.3(i). For $f \in E_{\alpha}^{A(\cdot)}$, we define $(J f)(t)=J(t) f(t) \in X_{\alpha-1}^{C(t)}$. Lemma 3.3(i) yields $\|J f\|_{\alpha-1}^{C(\cdot)} \leq c\|f\|_{\alpha-1}^{A(\cdot)}$ and the injectivity of $J$. There are $f_{n} \in E_{\alpha}^{A(\cdot)}$ converging to $f$ in $E_{\alpha-1}^{A(\cdot)}$ as $n \rightarrow \infty$. Then $J f_{n} \in E_{\alpha}^{A(\cdot)}$ and $J f_{n} \rightarrow J f$ with respect to $\|\cdot\|_{\alpha-1}^{C(\cdot)}$, and so $J f \in E_{\alpha-1}^{C(\cdot)}$. Thus assertion (i) holds. Assertion (ii) is a consequence of Proposition 3.4 and an interpolation argument.

Under the assumptions of assertion (iv), we take $u \in D\left(G_{\alpha-1}\right)$ and set $f=G_{\alpha-1} u \in$ $E_{\alpha-1}:=E_{\alpha-1}^{A(\cdot)}$. The equation

$$
u(t)=U(t, r) u(r)+\int_{r}^{t} U_{\alpha-1}(t, \tau) f(\tau) d \tau, \quad t>r,
$$

and the estimate (2.14) show that $\|u(t)\|_{\alpha-\epsilon}^{t} \leq c$ for all $t \in \mathbb{R}$ and some $\epsilon \in(0, \alpha-\beta)$. By interpolation with $u \in C_{0}(\mathbb{R}, X)$, it follows that $\|u(t)\|_{\beta}^{t} \rightarrow 0$ as $|t| \rightarrow \infty$. Thus $g_{n}(t):=R\left(\omega, A_{-1}(t)\right) B(t) n R(n, A(t)) u(t)$ tends to 0 in $X$ as $|t| \rightarrow \infty$, where $n \in \mathbb{N}$ is sufficiently large. Moreover, $g_{n}$ is continuous in $X$ by Remark 3.5(iv). The assumptions on $B(t)$ further imply that $g_{n}$ converges in $E$ to $g=R\left(\omega, A_{-1}(\cdot)\right) B(\cdot) u$ as $n \rightarrow \infty$ and that $\|g(t)\|_{\gamma}^{t} \leq c$ for $t \in \mathbb{R}$. Hence, Corollary 2.2.3 in [15] implies that $v \in E_{\alpha}$, and thus $B(\cdot) u \in E_{\alpha-1}$. Since $B(t) \in \mathcal{L}\left(X_{\beta}^{t}, X_{\alpha-1}^{t}\right)$ is uniformly bounded, we can establish formula (3.22) for $x \in X_{\alpha-1}^{s}$ as in the proof of Theorem 3.7. Using (3.22), Proposition 2.1 and Lemma 3.3, we compute

$$
\begin{aligned}
u(t)= & U(t, s) u(s)+\int_{s}^{t} U_{\alpha-1}(t, \tau) f(\tau) d \tau \\
= & U_{B}(t, s) u(s)+\int_{s}^{t} U_{B, \alpha-1}(t, \tau) f(\tau) d \tau-\int_{s}^{t} U_{B, \alpha-1}(t, \tau) B(\tau) U(\tau, s) u(s) d \tau \\
& \quad-\int_{s}^{t} \int_{\tau}^{t} U_{B, \alpha-1}(t, \sigma) B(\sigma) U_{\alpha-1}(\sigma, \tau) f(\tau) d \sigma d \tau .
\end{aligned}
$$

Similarly as in the proof of Theorem 3.7 one checks that the integrands of the last integral is measurable in $X$ for $s<\tau<\sigma<t$. Thus Fubini's theorem yields

$$
u(t)=U_{B}(t, s) u(s)+\int_{s}^{t} U_{B, \alpha-1}(t, \tau)(f(\tau)-B(\tau) u(\tau)) d \tau, \quad t \geq s, t, s \in \mathbb{R},
$$

so that $u \in D\left(G_{B, \alpha-1}\right)$ and $G_{B, \alpha-1} u=G_{\alpha-1} u-B(\cdot) u$. The converse inclusion is shown in the same way taking into account part (ii). Assertion (iii) is proved similarly as assertion (iv), using in addition part (i) and Lemma 3.3(i) in the second part.

\section{Robustness of exponential Dichotomy and Fredholmity}

Assume that (H2) holds. We want to show that the evolution family $U_{B}(\cdot, \cdot)$ generated by the operators $C(t)=\left(A_{\alpha-1}(t)+B(t)\right) \mid X, t \in \mathbb{R}$, inherits the exponential dichotomy or Fredholm properties of the evolution family $U(\cdot, \cdot)$ generated by $A(\cdot)$ if the perturbations $B(t), t \in \mathbb{R}$, are small in norm or compact. As a preparation, we note that formula (3.21), Proposition 2.1, Lemma 3.3 and Proposition 3.6 imply the crucial estimate

$$
\left\|U_{B}(s+1, s)-U(s+1, s)\right\|_{\mathcal{L}(X)} \leq c \sup _{\substack{s \leq t \leq s+1 \\ 14}}\|B(t)\|_{\mathcal{L}\left(X_{\alpha}^{t}, X_{\alpha-1}^{t}\right)} \int_{0}^{1}(1-\tau)^{\alpha-1} \tau^{-\alpha} d \tau
$$




$$
\leq c \sup _{s \leq t \leq s+1}\|B(t)\|_{\mathcal{L}\left(X_{\alpha}^{t}, X_{\alpha-1}^{t}\right)}
$$

for $s \in \mathbb{R}$, where $c$ only depends on the constants in (H2). Our first theorem on exponential dichtomies is an immediate consequence of (4.1) and Theorem 4.1 of [18], where we set

$$
\|B(\cdot)\|_{\infty}:=\sup _{t \in \mathbb{R}}\|B(t)\|_{\mathcal{L}\left(X_{\alpha}^{t}, X_{\alpha-1}^{t}\right)} .
$$

Theorem 4.1. Assume that (H2) holds and that $U(\cdot, \cdot)$ has an exponential dichotomy on a closed interval $J$ of $\mathbb{R}$. If $\|B(\cdot)\|_{\infty}$ is sufficiently small, then $U_{B}(\cdot, \cdot)$ has an exponential dichotomy on $J$ and the unstable projections of $U(\cdot, \cdot)$ and $U_{B}(\cdot, \cdot)$ have the same rank.

We next investigate the Fredholmity of the operator $G_{B, \alpha-1}$ defined in (3.25) which will lead to a Fredholm alternative for the equation $(3.23)$ on $J=\mathbb{R}$. To that purpose, we introduce the stable and unstable subspaces of $U_{B}(\cdot, \cdot)$ at $t_{0} \in \mathbb{R}$ by setting

$$
\begin{aligned}
X_{s}^{B}\left(t_{0}\right) & :=\left\{x \in X: \lim _{t \rightarrow+\infty}\left\|U_{B}\left(t, t_{0}\right) x\right\|=0\right\}, \\
X_{u}^{B}\left(t_{0}\right):=\left\{x \in X: \exists \text { a mild solution } u \in C_{0}\left(\left(-\infty, t_{0}\right], X\right) \text { of }(3.23)-(3.24)\right. & \text { on } \left.J=\left(-\infty, t_{0}\right] \text { with } f=0\right\} .
\end{aligned}
$$

(The above definition of $X_{s}^{B}\left(t_{0}\right)$ slightly differs from that of [16], but this fact does not play a role below.) Assume that $U_{B}(\cdot, \cdot)$ has an exponential dichotomy on $(-\infty,-T]$ and on $[T, \infty)$ for some $T \geq 0$. Theorem 3.6 and Remark 3.12 of [16] then yield the following results.

(a) $G_{B, \alpha-1}$ has closed range in $E_{\alpha-1}^{C(\cdot)}$ if and only if $X_{s}^{B}(T)+X_{u}^{B}(T)$ is closed in $X$.

(b) If (a) holds, then $\operatorname{codim} R\left(G_{B, \alpha-1}\right)=\operatorname{codim}\left(X_{s}^{B}(T)+X_{u}^{B}(T)\right)$.

(c) $\operatorname{dim} N\left(G_{B, \alpha-1}\right)=\operatorname{dim} X_{s}^{B}(T) \cap X_{u}^{B}(T)+\operatorname{dim} N\left(U_{B}(T,-T) \mid X_{u}^{B}(-T)\right)$.

The analogous results hold for $G$ and $G_{B}$ in $E$, and for $G_{\alpha-1}$ in $E_{\alpha-1}^{A(\cdot)}$. We further define the maps

$$
D x=\left(x_{n}-U(n, n-1) x_{n-1}\right)_{n \in \mathbb{Z}} \quad \text { and } \quad D_{B} x=\left(x_{n}-U_{B}(n, n-1) x_{n-1}\right)_{n \in \mathbb{Z}}
$$

for sequences $x=\left(x_{n}\right) \in c_{0}(\mathbb{Z}, X)$. Clearly, $D$ and $D_{B}$ are bounded opeators in $c_{0}(\mathbb{Z}, X)$. Due to Theorem 1.4 of [14], the operator $G$ (resp. $G_{B}$ ) on $E$ is Fredholm if and and only if $D$ (resp. $\left.D_{B}\right)$ is Fredholm on $c_{0}(\mathbb{Z}, X)$ and then ind $D=\operatorname{ind} G$ (resp. ind $D_{B}=\operatorname{ind} G_{B}$ ). (We remark that in [14] it was assumed that $(t, s) \longmapsto U(t, s)$ is strongly continuous at $t=s$, but the proof of Theorem 1.4 in [14] also works in our situation.) Moreover, in the case that the domains $D(A(t))$ are all dense in $X$ it was shown in [13, Theorem 1.1] that $U(\cdot, \cdot)$ has exponential dichotomies on $(-\infty,-T]$ and $[T, \infty)$ (for some $T \geq 0$ ) if $G$ is Fredholm in $E$. In the following result we can thus replace the assumption on the dichotomies by the condition that $A(t)$ is densely defined for all $t \in \mathbb{R}$.

Theorem 4.2. Assume that (H2) holds, that $G$ is Fredholm on $E$, and that $U(\cdot, \cdot)$ has an exponential dichotomy in $X$ on $(-\infty,-T]$ and on $[T, \infty)$ for some $T \geq 0$. Let $\|B(\cdot)\|_{\infty}$ be sufficiently small. Then $U_{B}(\cdot, \cdot)$ has an exponential dichotomy in $X$ on $(-\infty,-T]$ and on $[T, \infty)$, and $G_{B, \alpha-1}$ is Fredholm in $E_{\alpha-1}^{C(\cdot)}$ with the index $\operatorname{ind}\left(G_{B, \alpha-1}\right)=\operatorname{ind}(G)$.

Proof. Since $G$ is Fredholm on $E$, the operator $D$ is Fredholm on $c_{0}(\mathbb{Z}, X)$ with the same index due to [14, Theorem 1.4]. Estimate (4.1) and Theorem IV.5.22 of [11] then imply that $D_{B}$ is Fredholm on $c_{0}(\mathbb{Z}, X)$ with the same index provided that $\|B(\cdot)\|_{\infty}$ is smaller than a certain number $b>0$. Again by [14, Theorem 1.4], the operator $G_{B}$ is thus 
Fredholm on $E$ with the same index. Possibly after decrasing $b>0$, we deduce from Theorem 4.1 that $U_{B}(\cdot, \cdot)$ has exponential dichotomies on $(-\infty,-T]$ and $[T, \infty)$. Thus [16, Theorem 3.6] shows that $X_{s}^{B}(T)+X_{u}^{B}(T)$ is closed in $X$ and has codimension equal to $\operatorname{codim} R\left(G_{B}\right)$ and that

$$
\operatorname{dim}\left(X_{s}^{B}(T) \cap X_{u}^{B}(T)\right)+\operatorname{dim} N\left(U_{B}(T,-T) \mid X_{u}^{B}(-T)\right)=\operatorname{dim} N\left(G_{B}\right)<\infty .
$$

The assertion then follows from [16, Theorem 3.6].

Theorem 4.3. Assume that (H2) holds, that $A(t)$ is densely defined for every $t \in \mathbb{R}$, that $B(t): X_{\alpha}^{A(t)} \longrightarrow X_{\alpha-1}^{A(t)}$ is compact for every $t \in \mathbb{R}$, and that $\|B(t)\|_{\mathcal{L}\left(X_{\alpha}^{t}, X_{\alpha-1}^{t}\right)} \longrightarrow 0$ as $|t| \rightarrow \infty$. Let $G$ be Fredholm in $E$. Then the operator $G_{B, \alpha-1}$ is Fredholm in $E_{\alpha-1}^{C(\cdot)}$ with the index $\operatorname{ind}\left(G_{B, \alpha-1}\right)=\operatorname{ind}(G)$.

Proof. Due to our assumptions and [14, Theorem 1.4], the operator $D$ is Fredholm in $c_{0}(\mathbb{Z}, X)$ with index ind $G$. For $x=\left(x_{n}\right)_{n \in \mathbb{Z}} \in c_{0}(\mathbb{Z}, X)$ we define

$$
S x=\left(\int_{n-1}^{n} U_{\alpha-1}(n, \tau) B(\tau) U_{B}(\tau, n-1) x_{n-1} d \tau\right)_{n \in \mathbb{Z}} .
$$

Then $D_{B}=D-S$ by $(3.21)$, and thus $S \in \mathcal{L}\left(c_{0}(\mathbb{Z}, X)\right)$. We want to show that $S$ is compact. Take $\epsilon>0$ and $x=\left(x_{n}\right) \in c_{0}(\mathbb{Z}, X)$ with $\|x\| \leq 1$. For $z \in X$ and $n \in \mathbb{Z}$, we set

$$
K_{n} z:=\int_{n-1}^{n} U_{\alpha-1}(n, \tau) B(\tau) U_{B}(\tau, n-1) z d \tau \text {. }
$$

The operators $K_{n}$ converge to 0 in $\mathcal{L}(X)$ as $|n| \rightarrow \infty$ because of (4.1) and since $\|B(t)\|_{\mathcal{L}\left(X_{\alpha}^{t}, X_{\alpha-1}^{t}\right)} \rightarrow 0$ as $|t| \rightarrow \infty$. So there exists an index $N>0$ such that $\left\|K_{n} x_{n-1}\right\| \leq \epsilon$ for all $|n| \geq N$. On the other hand, as seen in the proof of Theorem 3.7 the map $\tau \longmapsto G_{n}(\tau) z:=U_{\alpha-1}(n, \tau) B(\tau) U_{B}(\tau, n-1) z \in X$ is continuous on $(n-1, n)$, and $\left\|G_{n}\right\| \in L^{1}(n-1, n)$. Moreover, the operator $G_{n}(\tau)$ is compact in $X$ for $\tau \in(n-1, n)$, due to the compactness of $B(\tau)$. Using Theorem C.7 of [8], we thus deduce that $K_{n}$ is compact in $X$ for each $n \in \mathbb{Z}$. This means that the set $\left\{K_{n} z: z \in X,\|z\| \leq 1, n \in\{-N, \cdots, N\}\right\}$ is contained in a compact set $K \subseteq X$. Therefore $S$ is compact.

Theorem IV.5.26 of [11] now shows that the operator $D_{B}$ is Fredholm on $c_{0}(\mathbb{Z}, X)$ with the index ind $G$. Hence, $G_{B}$ is Fredholm on $E$ with the same index by [14, Theorem 1.4]. Moreover, $U_{B}$ has exponential dichotomies on $(-\infty,-T]$ and $[T,+\infty)$ for some $T \geq 0$ due to [13, Theorem 1.1]. (Here we need the density of the domains.) The assertions then follow from [16, Theorem 3.6] as in the previous proof.

In the next remark we collect sufficient conditions for a part of hypothesis (H2) used in the above theorems.

Remark 4.4. Assume that the operators $A(t), t \in \mathbb{R}$, satisfy (2.1) and (2.2). Then the following assertions hold.

(i) Suppose either that for some $\beta \in[0, \alpha)$ the operators $B(t): X_{\beta}^{t} \longrightarrow X_{\alpha-1}^{t}$ are uniformly bounded for $t \in \mathbb{R}$ or that for some $\beta \in(\alpha, 1]$ the operators $B(t): X_{\alpha}^{t} \longrightarrow X_{\beta-1}^{t}$ are uniformly bounded for $t \in \mathbb{R}$. Then (H1) holds for $A(t)$ and $B(t)$ with a constant $\bar{\omega} \geq \omega$ for every $t \in \mathbb{R}$.

(ii) Suppose that the spaces $X_{\alpha}^{t}, t \in \mathbb{R}$, are isomorphic to a space $X_{\alpha}$ with uniformly equivalent norms. Assume that $B(t): X_{\alpha} \longrightarrow X_{\alpha-1}^{t}$ is compact, that $\|B(t)\|_{\mathcal{L}\left(X_{\alpha}, X_{\alpha-1}^{t}\right)} \rightarrow$ 0 as $|t| \rightarrow \infty$ and that $R\left(\omega, A_{-1}(\cdot)\right) B(\cdot) \in \mathcal{L}\left(X_{\alpha}\right)$ is locally Hölder continuous with exponent $\mu$. Then (H2) holds. 
Proof. Assertion (i) follows from Remark 3.1. For assertion (ii) we have to show that $A(t)$ and $B(t)$ satisfy (H1)(a) for $t \in \mathbb{R}$ with the same $\bar{\omega}$. Let $q=\left(2 c_{0}\right)^{-1}$ be given as in (H1). Let $\hat{c}$ be the constant for the equivalence of the norms of $X_{\alpha}^{t}$ and $X_{\alpha}$. Take $\bar{\omega} \geq \omega, t \in \mathbb{R}$, and $x \in X_{\alpha}$. Using (2.5) and choosing a sufficiently large $T_{0} \geq 0$, we first estimate

$$
\left\|R\left(\bar{\omega}, A_{-1}(t)\right) B(t) x\right\|_{\alpha}^{t} \leq(1+K)\|B(t)\|_{\mathcal{L}\left(X_{\alpha}, X_{\alpha-1}^{t}\right)} \hat{c}\|x\|_{\alpha}^{t} \leq q\|x\|_{\alpha}^{t}
$$

for $|t| \geq T_{0}$. Here $T_{0}$ does not depend on $\bar{\omega}$. Fix $\delta>0$ such that

$$
\hat{c}^{2}(1+K) \ell_{0} \delta^{\mu} \leq \frac{q}{4}
$$

where $\ell_{0}$ is the Hölder constant of $R\left(\omega, A_{-1}(\cdot)\right) B(\cdot)$ on $\left[-T_{0}, T_{0}\right]$. We fix a partition $-T_{0}=t_{0}<t_{1}<\cdots<t_{N}=T_{0}$ with $t_{k}-t_{k-1} \leq \delta$. Set $\epsilon=q(2 \hat{c}(1+K))^{-2}$. Since the operators $B(t)$ are compact, there exist vectors $y_{1 k}, \ldots, y_{m_{k} k} \in X_{\alpha-1}^{t_{k}}$ such that for each $x \in X_{\alpha}$ with $\|x\|_{\alpha} \leq \hat{c}$ there is an index $j=j(k, x)$ with

$$
\left\|B\left(t_{k}\right) x-y_{j k}\right\|_{\alpha-1}^{t_{k}} \leq \epsilon .
$$

Let $c_{y}$ be the maximum of all the norms $\left\|y_{j k}\right\|_{\alpha-1}^{t_{k}}$. Take $t \in\left[-T_{0}, T_{0}\right]$ and $x \in X_{\alpha}$ with $\|x\|_{\alpha}^{t} \leq 1$. Using (2.5), (2.4), (4.3), the Hölder continuity of $R\left(\omega, A_{-1}(\cdot)\right) B(\cdot)$ and (4.2), we obtain

$$
\begin{aligned}
& \left\|R\left(\bar{\omega}, A_{-1}(t)\right) B(t) x\right\|_{\alpha}^{t} \\
& \leq\left\|(\omega-A(t)) R(\bar{\omega}, A(t))\left[R\left(\omega, A_{-1}(t)\right) B(t)-R\left(\omega, A_{-1}\left(t_{k}\right)\right) B\left(t_{k}\right)\right] x\right\|_{\alpha}^{t} \\
& +\left\|(\omega-A(t)) R(\bar{\omega}, A(t)) R\left(\omega, A_{-1}\left(t_{k}\right)\right)\left[B\left(t_{k}\right) x-y_{j_{k}}\right]\right\|_{\alpha}^{t} \\
& +\left\|(\omega-A(t)) R(\bar{\omega}, A(t)) R\left(\omega, A_{-1}\left(t_{k}\right)\right) y_{j_{k}}\right\|_{\alpha}^{t} \\
& \leq \hat{c}(1+K)\left\|R\left(\omega, A_{-1}(t)\right) B(t) x-R\left(\omega, A_{-1}\left(t_{k}\right)\right) B\left(t_{k}\right) x\right\|_{\alpha}+\hat{c}^{2}(1+K)^{2}\left\|B\left(t_{k}\right) x-y_{j k}\right\|_{\alpha-1}^{t_{k}} \\
& +\frac{\hat{c}^{2} \omega K(1+K)}{1+\bar{\omega}-\omega}\left\|y_{j k}\right\|_{\alpha-1}^{t_{k}}+\left\|A(t) R(\bar{\omega}, A(t)) R\left(\omega, A_{-1}\left(t_{k}\right)\right) y_{j k}\right\|_{\alpha}^{t} \\
& \leq \hat{c}^{2}(1+K) \ell_{0} \delta^{\mu}+\frac{q}{4}+\frac{\hat{c}^{2} \omega K(1+K) c_{y}}{1+\bar{\omega}-\omega}+\hat{c}\left\|A(t) R(\bar{\omega}, A(t)) R\left(\omega, A_{-1}\left(t_{k}\right)\right) y_{j k}\right\|_{\alpha} \\
& \leq q
\end{aligned}
$$

for sufficiently large $\bar{\omega}$ independent of $t$. (Observe that $A(\cdot) R(\bar{\omega}, A(\cdot))$ converges in $C\left(\left[-T_{0}, T_{0}\right], X_{\alpha}\right)=: F$ to 0 as $\bar{\omega} \rightarrow \infty$ since the part of the multiplication operator $A(\cdot)$ in $F$ is sectorial.)

Using Theorems 4.2 and 4.3 as well as [13, Theorem 1.1] and (the proof of) [16, Theorem 3.10], we finally obtain the following Fredholm alternative for the perturbed evolution equation

$$
u^{\prime}(t)=A_{-1}(t) u(t)+B(t) u(t)+f(t), \quad t \in \mathbb{R} .
$$

Below we use the extensions to $X_{\alpha-1}^{C(t)}$ of the dichotomy propjections $Q_{B}(t)$ and $P_{B}(t)=$ $I-Q_{B}(t)$ of $U_{B}(\cdot, \cdot)$, cf. [16, Prop.2.2].

Theorem 4.5. Assume that the assumptions of Theorem 4.2 or 4.3 hold. Let $f \in E_{\alpha-1}^{C(\cdot)}$. Then there is a mild solution $u \in C_{0}(\mathbb{R}, X)$ of (4.4) if and only if

$$
\int_{\mathbb{R}}\langle f(s), w(s)\rangle_{X_{\alpha-1}^{C(s)}} d s=0
$$


for each $w \in L^{1}\left(\mathbb{R}, X^{*}\right)$ with $w(s)=U_{B, \alpha-1}(t, s)^{*} w(t)$ for all $t \geq s$. The mild solutions $u$ are given by

$$
\begin{aligned}
& u(t)=v(t)-U_{B}(t, T) y_{s}+\left(R_{\alpha-1}^{+} f\right)(t), \quad t \geq T, \\
& u(t)=v(t)+\tilde{v}(t)+\left(R_{\alpha-1}^{-} f\right)(t), \quad t \leq T
\end{aligned}
$$

where $\left(R_{\alpha-1}^{+} f\right)(T)-\left(R_{\alpha-1}^{-} f\right)(T)=y_{s}+y_{u} \in X_{s}^{B}(T)+X_{u}^{B}(T), \tilde{v} \in C_{0}((-\infty, T], X)$ with $\tilde{v}(T)=y_{u}$ and $\tilde{v}(t)=U_{B}(t, s) \tilde{v}(s)$ for all $T \geq t \geq s$, and $v \in C_{0}(\mathbb{R}, X)$ with $v(t)=U_{B}(t, s) v(s)$ for all $t \geq s$. Finally, the functions $R_{\alpha-1}^{ \pm} f$ are defined by

$$
\begin{aligned}
& R_{\alpha-1}^{+} f(t)=\int_{T}^{t} U_{B, \alpha-1}(t, s) P_{B, \alpha-1}(s) f(s) d s-\int_{t}^{\infty} \widetilde{U}_{B, \alpha-1}(t, s) Q_{B, \alpha-1}(s) f(s) d s, \quad t \geq T, \\
& R_{\alpha-1}^{-} f(t)=\int_{-\infty}^{t} U_{B, \alpha-1}(t, s) P_{B, \alpha-1}(s) f(s) d s-\int_{t}^{-T} \widetilde{U}_{B, \alpha-1}(t, s) Q_{B, \alpha-1}(s) f(s) d s, \quad t \leq-T, \\
& R_{\alpha-1}^{-} f(t)=\int_{-\infty}^{-T} U_{B, \alpha-1}(t, s) P_{B, \alpha-1}(s) f(s) d s+\int_{-T}^{t} U_{B, \alpha-1}(t, s) f(s) d s, \quad-T \leq t \leq T .
\end{aligned}
$$

\section{Perturbed boundary parabolic evolution equations}

In this section we study the inhomogeneous perturbed boundary evolution problem

$$
\left\{\begin{array}{l}
u^{\prime}(t)=A_{m}(t) u(t)+g(t), \quad t \in \mathbb{R}, \\
L(t) u(t)=\Phi(t) u(t)+h(t), \quad t \in \mathbb{R},
\end{array}\right.
$$

on Banach spaces $X$ and $Y$, where $g \in C_{0}(\mathbb{R}, X)$ and $h \in C_{0}(\mathbb{R}, Y)$ are given. We introduce our hypotheses.

(A1) For every $t \in \mathbb{R}$ there is a Banach space $Z_{t} \hookrightarrow X$ and operators $A_{m}(t) \in \mathcal{L}\left(Z_{t}, X\right)$ and $L(t) \in \mathcal{L}\left(Z_{t}, Y\right)$ such that the restrictions $A(t)$ of $A_{m}(t)$ to $N(L(t)), t \in \mathbb{R}$, satisfy (2.1) and (2.2) with constants $\omega \in \mathbb{R}, \phi \in(\pi / 2, \pi), K, L \geq 0$, and $\mu, \nu \in$ $(0,1]$. For some $\alpha \in(1-\mu, 1)$ and every $t \in \mathbb{R}$, we have $Z_{t} \hookrightarrow X_{\alpha}^{t}$ with a uniform embedding constant, and there are operators $\Phi(t) \in \mathcal{L}\left(X_{\alpha}^{t}, Y\right)$.

Let (A1) hold. As recalled in Section 2, the operators $A(\cdot)$ then generate an evolution family $U(\cdot, \cdot)$ on $X$. We recall another concept used below. Let $\hat{\omega} \in \mathbb{R}$. We say that the abstract boundary value problem

$$
\left(\hat{\omega}-A_{m}(t)\right) v=0, \quad L(t) v=\varphi,
$$

is well posed with solution operator $D(t)$ if $D(t) \in \mathcal{L}\left(Y, Z_{t}\right)$ and, for each $\varphi \in Y$, there exists a unique solution $v \in Z_{t}$ given by $v=D(t) \varphi$.

(A2) For some $\hat{\omega} \geq \omega$ and each $t \in \mathbb{R}$, there exists a solution operator $D(t) \in \mathcal{L}\left(Y, Z_{t}\right)$ of (5.2) such that $D(t): Y \longrightarrow X_{\alpha}^{t}$ is uniformly bounded and

$$
\|D(t) \Phi(t)\|_{\mathcal{L}\left(X_{\alpha}^{t}\right)} \leq q:=\frac{1}{2 c_{0}}=\frac{1}{2}\left[1+\frac{K}{\sin (\pi-\phi)}\right]^{-1}, \quad t \in \mathbb{R} .
$$

(A3) There is a Banach space $X_{\alpha}$ such that $X_{\alpha}^{t} \hookrightarrow X_{\alpha} \hookrightarrow X$ with a uniform embedding constant and $\|D(t) \Phi(t) x-D(s) \Phi(s) x\|_{\alpha}^{t} \leq c \mid t-s^{\mu}\|x\|_{\alpha}$ for all $x \in X_{\alpha}$ and $t, s \in \mathbb{R}$. If $\alpha>\nu$, we assume in addition that the norms of $X_{\alpha}$ and $X_{\alpha}^{t}$ are uniformly equivalent for $t \in \mathbb{R}$ and that the map $\mathbb{R} \ni t \longmapsto R(\omega, A(\cdot)) \in \mathcal{L}\left(X, X_{\alpha}\right)$ is uniformly Hölder continuous with exponent $\mu$. 
Remark 5.1. (i) The problem (5.2) is well posed if (A1) holds and $L(t)$ is surjective, see [9, Lemma 1.2].

(ii) If the problem (5.2) is well posed for some $\hat{\omega} \in \mathbb{R}$ with solution operator $D(t)$, then it is well posed for every $\mu \in \rho(A(t))$ with the solution operator $D_{\mu}(t):=[I-(\mu-$ $\hat{\omega}) R(\mu, A(t))] D(t)$. In fact, the uniqueness of solutions follows from the injectivity of the operator $\mu-A(t)=\left(\mu-A_{m}(t)\right) \mid N(L(t))$. Moreover, the operator $D_{\mu}(t)$ is bounded from $Y$ into $Z_{t}$. Finally, for $\varphi \in Y$ we have

$$
\begin{aligned}
\left(\mu-A_{m}(t)\right) D_{\mu}(t) \varphi & =\left(\mu-A_{m}(t)\right) D(t) \varphi-(\mu-\hat{\omega}) D(t) \varphi=0 \\
L(t) D_{\mu}(t) \varphi & =L(t) D(t) \varphi-(\mu-\hat{\omega}) L(t) R(\mu, A(t))) D(t)=\varphi .
\end{aligned}
$$

(iii) In (ii) one further has $R(\hat{\omega}, A(t)) D_{\mu}(t)=R(\mu, A(t)) D(t)$, due to the resolvent equation.

As in [16], we can rewrite (5.1) as the inhomogeneous perturbed evolution equation

$$
u^{\prime}(t)=A_{\alpha-1}(t) u(t)+B(t) u(t)+f(t), \quad t \in \mathbb{R},
$$

where $f(t):=g(t)+\left(\omega-A_{\alpha-1}(t)\right) D_{\omega}(t) h(t)$ and

$$
B(t):=\left(\omega-A_{\alpha-1}(t)\right) D_{\omega}(t) \Phi(t), \quad t \in \mathbb{R} .
$$

The mild solutions of (5.3) are said to be mild solutions of (5.1). We first investigate the well posedness and the asymptotic behavior of the Cauchy problem corresponding (5.1), where $g=h=0$. Here we start with generation properties of the operators

$$
A_{\Phi}(t):=A_{m}(t) \quad \text { with } \quad D\left(A_{\Phi}(t)\right)=\left\{x \in Z_{t}: L(t) x=\Phi(t) x\right\}, \quad t \in \mathbb{R} .
$$

In some results we can replace (A1) by the following somewhat weaker assumption.

(A1') For every $t \in \mathbb{R}$ there is a Banach space $Z_{t} \hookrightarrow X$ and operators $A_{m}(t) \in \mathcal{L}\left(Z_{t}, X\right)$ and $L(t) \in \mathcal{L}\left(Z_{t}, Y\right)$ such that the restrictions $A(t)$ of $A_{m}(t)$ to $N(L(t)), t \in \mathbb{R}$, satisfy (2.1) with constants $\omega \in \mathbb{R}, \phi \in(\pi / 2, \pi)$ and $K \geq 0$. For some $\alpha \in(0,1)$ and every $t \in \mathbb{R}$, we have $Z_{t} \hookrightarrow X_{\alpha}^{t}$ with a uniform embedding constant, and there are operators $\Phi(t) \in \mathcal{L}\left(X_{\alpha}^{t}, Y\right)$.

Proposition 5.2. If (A1') and (A2) hold, then the following assertions are true for $t \in \mathbb{R}$.

(i) The operators $A(t)$ and $B(t)$ satisfy (H1) with uniform constants and $A_{\Phi}(t)$ satisfies condition (2.1) with constants $\hat{\omega}, \phi$, and some $\hat{K}$ depending on $K, \phi, \omega, \hat{\omega}$. Moreover, $A_{\Phi}(t)$ is the part $C(t)$ of $A_{\alpha-1}(t)+B(t)$ in $X$.

(ii) $X_{\alpha}^{A(t)} \hookrightarrow X_{\alpha}^{A_{\Phi}(t)}$ and $X_{\alpha-1}^{A(t)} \hookrightarrow X_{\alpha-1}^{A_{\Phi}(t)}$, where the norms of the embeddings are bounded by constants only depending on the constants in (A1') and (A2).

(iii) $X_{\alpha+\epsilon}^{A_{\Phi}(t)} \hookrightarrow X_{\alpha}^{A}(t)$ for $0<\epsilon<1-\alpha$, where the norms of the embeddings are bounded by constants only depending on $\epsilon$ and the constants in (A1') and (A2).

Proof. Remark 5.1 implies that

$$
R\left(\hat{\omega}, A_{-1}(t)\right) B(t)=\left(\omega-A_{\alpha-1}(t)\right) R\left(\omega, A_{-1}(t)\right) D(t) \Phi(t)=D(t) \Phi(t) .
$$

Due to (5.4) and (A2), the operators $B(t)$ are uniformly bounded from $X_{\alpha}^{t}$ to $X_{\alpha-1}^{t}$ for $t \in \mathbb{R}$, and the estimate (H1)(a) holds with $\bar{\omega}=\hat{\omega}$. If $x \in D\left(A_{\Phi}(t)\right) \hookrightarrow X_{\alpha}^{t}$, then we have

$$
\begin{aligned}
A_{m}(t) x & =\left(A_{m}(t)-\omega\right)\left(x-D_{\omega}(t) L(t) x\right)+\omega x=(A(t)-\omega)\left(x-D_{\omega}(t) L(t) x\right)+\omega x \\
& =A_{\alpha-1}(t) x+\left(\omega-A_{\alpha-1}(t)\right) D_{\omega}(t) \Phi(t) x=A_{\alpha-1}(t) x+B(t) x .
\end{aligned}
$$


Hence, $x \in D(C(t))$ and $C(t) x=A_{\Phi}(t) x$, i.e, $A_{\Phi}(t) \subset C(t)$. For $x \in D(C(t))$, we first note that

$$
(C(t)-\omega) x=\left(A_{\alpha-1}(t)-\omega\right)\left(x-D_{\omega}(t) \Phi(t) x\right) \in X .
$$

Therefore $x-D_{\omega}(t) \Phi(t) x \in D(A(t))$. So we obtain $x \in Z_{t}$ and $0=L(t)\left(x-D_{\omega}(t) \Phi(t) x=\right.$ $L(t) x-\Phi(t) x$. As a result, $x \in D\left(A_{\Phi}(t)\right)$ and thus $A_{\Phi}(t)=C(t)$. Proposition 3.2 and Lemma 3.3 now imply the assertions.

The next result gives a suffient condition for (A2) and for the equality of the inter/extrapolation spaces of $A(t)$ and $A_{\Phi}(t)$.

Proposition 5.3. Assume that (A1') holds and that for some $\hat{\omega} \geq \omega$ and every $t \in \mathbb{R}$ there exists a solution operator $D(t)$ of (5.2) such that $d:=\sup _{t \in \mathbb{R}}\left\|D_{\omega}(t)\right\|_{\mathcal{L}\left(Y, X_{\alpha}^{t}\right)}<\infty$. Moreover, suppose that one of the following three conditions are true.

(a) $\|\Phi(t)\|_{\mathcal{L}\left(X_{\alpha}^{t}, Y\right)} \leq\left[4(1+K) c_{0} d\right]^{-1}$ for $t \in \mathbb{R}$.

(b) The operators $\Phi(t), t \in \mathbb{R}$, are uniformly bounded in $\mathcal{L}\left(X_{\beta}^{t}, Y\right)$ for some $\beta \in[0, \alpha)$.

(c) For $t \in \mathbb{R}$ we have $Z_{t} \hookrightarrow X_{\gamma}^{t}$ with uniformly bounded embedding constants and the operators $D_{\omega}(t)$ are uniformly bounded in $\mathcal{L}\left(Y, X_{\gamma}^{t}\right)$ for some $\gamma \in(\alpha, 1]$.

Then condition (A2) and thus assertion (i) of Proposition 5.2 hold. Moreover, we have $X_{\alpha}^{A(t)}=X_{\alpha}^{A_{\Phi}(t)}$ and $X_{\alpha-1}^{A(t)} \cong X_{\alpha-1}^{A_{\Phi}(t)}$, where the respective norms are equivalent with constants only depending on the given constants.

Proof. In the case $(\mathrm{a})$, we have $\|B(t)\|_{\mathcal{L}\left(X_{\alpha}^{t}, X_{\alpha-1}^{t}\right)} \leq\left[4(1+K) c_{0}\right]^{-1}$ for all $t \in \mathbb{R}$. In the case (b) and (c), one sees that $B(t)$ is uniformly bounded from $X_{\beta}^{t}$ to $X_{\alpha-1}^{t}$ and from $X_{\alpha}^{t}$ to $X_{\gamma-1}^{t}$, repectively. Thus (5.4), Remark 5.1, and Proposition 3.4 yield the results.

Proposition 5.4. Let (A1)-(A3) hold. Then the operators $A(t)$ and $B(t), t \in \mathbb{R}$, satisfy the hypothesis (H2), and the operators $A_{\Phi}(t), t \in \mathbb{R}$, fulfill the conditions (2.1) and (2.2) with exponents $\mu$ and $\min \{\nu, \alpha\}$.

Proof. Assumption (A3) combined with (5.4) implies (3.20) in (H2) for $A(t)$ and $B(t)$. The assertions thus follow from Propositions 5.2 and 3.6.

Thanks to the above proposition, the operators $A_{\Phi}(\cdot)$ generate an evolution family $U_{\Phi}(\cdot, \cdot)$ with the properties stated in Section 2. In particular, it can be extended to a family $U_{\Phi, \alpha-1}(t, s): X_{\alpha-1}^{\Phi(t)} \longrightarrow X$ which gives the mild solution of the boundary parabolic evolution equation (5.1). We write $G_{\Phi, \alpha-1}$ instead of $G_{B, \alpha-1}$. Moreover, Theorem 3.7 implies the following variation of constants formulas.

Proposition 5.5. Assume that (A1)-(A3) hold. Then, for $x \in X$ and $t \geq s$, we have

$$
\begin{aligned}
& U_{\Phi}(t, s) x=U(t, s) x+\int_{s}^{t} U_{\alpha-1}(t, \tau)\left(\omega-A_{\alpha-1}(\tau)\right) D_{\omega}(\tau) \Phi(\tau) U_{\Phi}(\tau, s) x d \sigma \\
& U_{\Phi}(t, s) x=U(t, s) x+\int_{s}^{t} U_{\Phi, \alpha-1}(t, \tau)\left(\omega-A_{\alpha-1}(\tau)\right) D_{\omega}(\tau) \Phi(\tau) U(\tau, s) x d \tau .
\end{aligned}
$$

From Theorems 4.1, 4.2, and 4.3 we further deduce the robustness results stated below. The compactness assumption in the second result holds in particular if $Z_{t}$ is compactly embedded in $X_{\alpha}^{t}$ for $t \in \mathbb{R}$ which typically holds in applications to partial differential equations on a bounded spatial domain. 
Theorem 5.6. Assume that (A1)-(A3) hold and that $\sup _{t \in \mathbb{R}}\|\Phi(\cdot)\|_{\mathcal{L}\left(X_{\alpha}^{t}, Y\right)}$ is sufficiently small. Then the following assertions are true.

(i) If $U$ has an exponential dichotomy on a closed interval $J$ of $\mathbb{R}$, then $U_{\Phi}$ has an exponential dichotomy on $J$ and the unstable projections of $U(\cdot, \cdot)$ and $U_{\Phi}(\cdot, \cdot)$ have the same rank.

(ii) If $U$ has exponential dichotomy on $(-\infty,-T]$ and $[T, \infty)$ for some $T \geq 0$ and $G$ is Fredholm on $E$, then $G_{\Phi, \alpha-1}$ is Fredholm on $E_{\alpha-1}^{A_{\Phi}(\cdot)}$ with the index $\operatorname{ind}\left(G_{\Phi, \alpha-1}\right)=\operatorname{ind}(G)$.

Theorem 5.7. Assume that (A1)-(A3) hold, that $A(t)$ is densely defined for every $t \in \mathbb{R}$, that $D(t) \Phi(t) \in \mathcal{L}\left(X_{\alpha}^{A(t)}\right)$ is compact for every $t \in \mathbb{R}$ and tends to 0 in norm as $|t| \rightarrow \infty$. Let $G$ be Fredholm in $E$. Then the operator $G_{\Phi, \alpha-1}$ is Fredholm in $E_{\alpha-1}^{A_{\Phi}(\cdot)}$ with the index $\operatorname{ind}\left(G_{\Phi, \alpha-1}\right)=\operatorname{ind}(G)$.

Theorem 4.5 now yields a Fredholm alternative for (5.1). We note that $D_{\omega}(\cdot) h \in E_{\alpha}$ due to the additional assumptions on $D(t)$, Remark 5.1(ii), and [15, Corollary 2.2.3]. We write $P_{\Phi}, Q_{\Phi}, X_{s}^{\Phi}(T)$, and $X_{u}^{\Phi}(T)$ instead of $P_{B}, Q_{B}, X_{s}^{B}(T)$, and $X_{u}^{B}(T)$.

Theorem 5.8. Assume that the assumptions of Theorem 5.6 or 5.7 hold. Let $g \in C_{0}(\mathbb{R}, X)$ and $h \in C_{0}(\mathbb{R}, Y)$. Suppose that $t \mapsto D(t) y \in X$ is continuous for each $y \in Y$ and that $D(t): Y \rightarrow X_{\beta}^{t}$ is uniformly bounded for some $\beta \in(\alpha, 1)$. Then there is a mild solution $u \in C_{0}(\mathbb{R}, X)$ of (5.1) if and only if

$$
\int_{\mathbb{R}}\langle f(s), w(s)\rangle_{X_{\alpha-1}^{A_{\Phi}(s)}} d s=0
$$

for $f=g+\left(\omega-A_{\alpha-1}(\cdot)\right) D_{\omega}(\cdot) h$ and all $w \in L^{1}\left(\mathbb{R}, X^{*}\right)$ with $w(s)=U_{\Phi, \alpha-1}(t, s)^{*} w(t)$ for all $t \geq s$. The mild solutions $u$ are given by

$$
\begin{aligned}
& u(t)=v(t)-U_{\Phi}(t, T) y_{s}+\left(R_{\alpha-1}^{+} f\right)(t), \quad t \geq T, \\
& u(t)=v(t)+\tilde{v}(t)+\left(R_{\alpha-1}^{-} f\right)(t), \quad t \leq T,
\end{aligned}
$$

where $\left(R_{\alpha-1}^{+} f\right)(T)-\left(R_{\alpha-1}^{-} f\right)(T)=y_{s}+y_{u} \in X_{s}^{\Phi}(T)+X_{u}^{\Phi}(T), \tilde{v} \in C_{0}((-\infty, T], X)$ with $\tilde{v}(T)=y_{u}$ and $\tilde{v}(t)=U_{\Phi}(t, s) \tilde{v}(s)$ for all $T \geq t \geq s$, and $v \in C_{0}(\mathbb{R}, X)$ with $v(t)=U_{\Phi}(t, s) v(s)$ for all $t \geq s$. Finally, $R_{\alpha-1}^{ \pm} f$ are defined by

$$
\begin{aligned}
& R_{\alpha-1}^{+} f(t)=\int_{T}^{t} U_{\Phi, \alpha-1}(t, s) P_{\Phi, \alpha-1}(s) f(s) d s-\int_{t}^{\infty} \widetilde{U}_{\Phi, \alpha-1}(t, s) Q_{\Phi, \alpha-1}(s) f(s), d s, \quad t \geq T, \\
& R_{\alpha-1}^{-} f(t)=\int_{-\infty}^{t} U_{\Phi, \alpha-1}(t, s) P_{\Phi, \alpha-1}(s) f(s) d s-\int_{t}^{-T} \widetilde{U}_{\Phi, \alpha-1}(t, s) Q_{\Phi, \alpha-1}(s) f(s) d s, \quad t \leq-T, \\
& R_{\alpha-1}^{-} f(t)=\int_{-\infty}^{-T} U_{\Phi, \alpha-1}(t, s) P_{\Phi, \alpha-1}(s) f(s) d s+\int_{-T}^{t} U_{\Phi, \alpha-1}(t, s) f(s) d s, \quad-T \leq t \leq T .
\end{aligned}
$$

We illustrate our results by an example in a sup norm context involving a nonlocal unbounded perturbation at the boundary.

Example 5.9. We study the boundary value problem

$$
\begin{aligned}
\partial_{t} u(t, x) & =A(t, x, D) u(t, x), \quad t \in \mathbb{R}, x \in \Omega, \\
L(t, x, D) u(t, x) & =(\Phi(t) u)(t, x), \quad t \in \mathbb{R}, x \in \partial \Omega,
\end{aligned}
$$

on a bounded domain $\Omega \subseteq \mathbb{R}^{n}$ with boundary $\partial \Omega$ of class $C^{2}$ and outer unit normal vector $\nu(x)$, employing the differential expressions

$$
A(t, x, D)=\sum_{k, l} a_{k l}(t, x) \partial_{k} \partial_{l}+\sum_{k} a_{k}(t, x) \partial_{k}+a_{0}(t, x)
$$




$$
L(t, x, D)=\sum_{k} b_{k}(t, x) \partial_{k}+b_{0}(t, x) .
$$

We require that $a_{k l}=a_{l k}$ and $b_{k}$ are real-valued, $a_{k l}, a_{k}, a_{0} \in C_{b}^{\mu}(\mathbb{R}, C(\bar{\Omega})), b_{k}, b_{0} \in$ $C_{b}^{\mu}\left(\mathbb{R}, C^{1}(\partial \Omega)\right)$,

$$
\sum_{k, l=1}^{n} a_{k l}(t, x) \xi_{k} \xi_{l} \geq \eta|\xi|^{2}, \quad \text { and } \quad \sum_{k=1}^{n} b_{k}(t, x) \nu_{k}(x) \geq \beta
$$

for constants $\mu \in(1 / 2,1), \beta, \eta>0$ and all $\xi \in \mathbb{R}^{n}, k, l=1, \cdots, n, t \in \mathbb{R}, x \in \bar{\Omega}$ resp. $x \in \partial \Omega$. ( $C_{b}^{\mu}$ is the space of bounded, globally Hölder continuous functions.) We set $X=C(\bar{\Omega}), Y=C^{1}(\partial \Omega)$,

$$
Z_{t}=\left\{u \in \bigcap_{p>1} W_{p}^{2}(\Omega): A(t, \cdot, D) u \in X, L(t, \cdot, D) u \in Y\right\},
$$

$A_{m}(t) u=A(t, \cdot, D) u$ and $L(t) u=L(t, \cdot, D) u$ for $u \in Z_{t}$, and $A(t)=A_{m}(t) \mid N(L(t))$.

As shown in $[3, \S 6]$ the operators $A(t), t \in \mathbb{R}$, satisfy (2.1) and (2.2) with $\nu=1 / 2$. (We note that by the same methods it can be shown that for all $p \in(1, \infty)$ the map $t \mapsto R(\omega, A(t)) \in \mathcal{L}\left(X, W^{2, p}(\Omega)\right)$ is Hölder continuous with exponent $\mu$, but this fact is not needed below.) In particular, $A(\cdot)$ generates an evolution family $U(\cdot, \cdot)$ on $X$. Observe that $D(A(t))$ is dense in $X$. We endow $Z_{t}$ with the norm $\|u\|_{Z_{t}}=\|u\|_{\infty}+\left\|A_{m}(t) u\right\|_{\infty}+$ $\|L(t) u\|_{Y}$. The completeness of this norm can be deduced from the elliptic $L^{p}$-apriori estimates, see e.g. [15, Theorem 3.1.1].

By standard elliptic theory, for each $\varphi \in Y$ there is a unique $v \in \bigcap_{p>1} W_{p}^{2}(\Omega)$ satisfying

$$
(\omega-A(t, \cdot, D)) v=0 \quad \text { on } \Omega, \quad L(t, \cdot, D) v=\varphi \quad \text { on } \partial \Omega,
$$

and $\|v\|_{2, p} \leq c_{p}\|\varphi\|_{Y}$, where $\omega \geq 0$ is sufficently large. For $p>n / 2$, Sobolev's embedding theorem yields $A(t, \cdot, D) v=\omega v \in X$ and thus $\|A(t, \cdot, D) v\|_{\infty} \leq c\|v\|_{2, p} \leq c\|\varphi\|_{Y}$. (Here and below all constants do not depend on $t$.) As a result, the solution operator $D_{\omega}(t) \varphi:=v$ is continuous from $Y$ to $Z_{t}$. In [16, Example 4.5] we have checked that $t \mapsto D_{\omega}(t) \in$ $\mathcal{L}\left(Y, W_{p}^{2}(\Omega)\right)$ is Hölder continuous of exponent $\mu$ for all $p \in(1, \infty)$.

For $\alpha \in(0,1 / 2)$, Theorem 3.1.30 in [15] shows that $X_{\alpha}^{t}=h^{2 \alpha}(\bar{\Omega})=: X_{\alpha}$, where the 'small Hölder space' $h^{\beta}$ is defined in $[15, \S 0.2]$. (Moreover, $X_{\alpha}^{t}$ is the kernel of $L(t)$ in $h^{2 \alpha}(\bar{\Omega})$ for $\alpha \in(1 / 2,1)$.) Thus $Z_{t}$ is compactly embedded in $X_{\alpha}^{t}$ for $\alpha \in(0,1 / 2)$ and $t \in \mathbb{R}$. We further assume that

$$
a_{\beta}(t, \cdot) \rightarrow a_{\beta}( \pm \infty, \cdot) \quad \text { in } X \quad \text { and } \quad b_{j}(t, \cdot) \rightarrow b_{j}( \pm \infty, \cdot) \quad \text { in } Y
$$

as $t \rightarrow \pm \infty$, where $\beta=(k, l)$ or $\beta=j$ for $k, l=1, \cdots, n$ and $j=0, \cdots, n$. Defining the sectorial operators $A_{ \pm \infty}$ in the same way as $A(t)$, we also suppose that $i \mathbb{R} \subset \rho\left(A_{ \pm \infty}\right)$. (Observe that the operators $A_{ \pm \infty}$ have compact resolvent so that the spectrum consists only of eigenvalues. The spectrum of $A_{ \pm \infty}$ was studied in [7, Exa.5.1].) Then $U(\cdot, \cdot)$ has exponential dichotomies on $(-\infty,-T]$ and $[T, \infty)$ and the operator $G$ is Fredholm on $E$ due to Theorem 4.3 of [18] and Corollary 3.7 and Example 4.5 of [16].

Now we fix $\alpha \in(1-\mu, 1 / 2)$. We assume that $\Phi(t) \in \mathcal{L}\left(X_{\alpha}, Y\right)$ is globally Hölder continuous of exponent $\mu$ and $\|\Phi(t)\|_{\mathcal{L}\left(X_{\alpha}, Y\right)} \longrightarrow 0$ as $|t| \rightarrow \infty$. For instance, one could take $\Phi(t)=\gamma m(t, \cdot)\left(I-\Delta_{N}\right)^{-\beta}$ for the Neumann Laplacian $\Delta_{N}$ on $X, \beta \in[0, \alpha]$, the trace operator $\gamma$ and a function $m \in C_{b}^{\mu}\left(\mathbb{R}, C^{1}(\bar{\Omega})\right) \cap C_{0}\left(\mathbb{R}, C^{1}(\bar{\Omega})\right)$. Remark 4.4 and (5.4) imply that the estimate in (A2) holds for a sufficiently large $\hat{\omega}=\bar{\omega}$. 
We define the mild solutions of (5.5) again by (5.1). Then the Fredholm alternative Theorem 5.8 holds for mild solutions of (5.5) on $X=C(\bar{\Omega})$ for $g \in C_{0}(\mathbb{R}, X)$ and $h \in$ $C_{0}(\mathbb{R}, Y)$.

\section{REFERENCES}

[1] P. Acquistapace, F. Flandoli and B. Terreni, Initial boundary value problems and optimal control for nonautonomous parabolic systems. SIAM J. Control Optim. 29 (1991), 89-118.

[2] P. Acquistapace and B. Terreni, A unified approach to abstract linear nonautonomous parabolic equations. Rend. Sem. Mat. Univ. Padova 78 (1987), 47-107.

[3] P. Acquistapace, Evolution operators and strong solutions of abstract linear parabolic equations. Differential Integral Equations 1 (1988), 433-457.

[4] H. Amann, Linear and Quasilinear Parabolic Problems. Volume 1: Abstract Linear Theory. Birkhäuser, Basel, 1995.

[5] C. Chicone and Y. Latushkin, Evolution Semigroups in Dynamical Systems and Differential Equations. American Mathematical Society, Providence (RI), 1999.

[6] D. Di Giorgio and A. Lunardi, On Fredholm properties of $\mathcal{L} u=u^{\prime}-A(t) u$ for paths of sectorial operators. Proc. Roy. Soc. Edinburgh Sect. A 135 (2005), 39-59.

[7] D. Di Giorgio, A. Lunardi and R. Schnaubelt, Fredholm properties of abstract parabolic operators in $L^{p}$ spaces on the real line. Proc. London Math. Soc. 91 (2005), 703-737.

[8] K.-J. Engel and R. Nagel, One-Parameter Semigroups for Linear Evolution Equations. SpringerVerlag, New York, 2000.

[9] G. Greiner, Perturbing the boundary conditions of a generator. Houston J. Math. 13 (1987), 213-229.

[10] B. Haak, M. Haase and P.C. Kunstmann, Perturbation, interpolation, and maximal regularity. Adv. Differential Equations 11 (2006), 201-240.

[11] T. Kato, Perturbation Theory for Linear Operators. Springer-Verlag, Berlin, 1980.

[12] P.C. Kunstmann and L. Weis, Perturbation theorems for maximal $L_{p}$-regularity. Ann. Scuola Norm. Sup. Pisa Cl. Sci. (4) 30 (2001), 415-435.

[13] Y. Latushkin, A. Pogan and R. Schnaubelt, Dichotomy and Fredholm properties of evolution equations. J. Operator Theory, to appear.

[14] Y. Latushkin and A. Tomilov, Fredholm differential operators with unbounded coefficients. J. Differential Equations 208 (2005), 388-429.

[15] A. Lunardi, Analytic Semigroups and Optimal Regularity in Parabolic Problems. Birkhäuser, Basel, 1995.

[16] L. Maniar and R. Schnaubelt, The Fredholm alternative for parabolic evolution equations with inhomogeneous boundary conditions. J. Differential Equations, 235 (2007), 308-339.

[17] R. Schnaubelt, Well-posedness and asymptotic behavior of non-autonomous linear evolution equations. In: A. Lorenzi and B. Ruf (Eds.), "Evolution Equations, Semigroups and Functional Analysis," Birkhäuser, Basel, 2002, pp. 311-338.

[18] R. Schnaubelt, Asymptotic behaviour of parabolic nonautonomous evolution equations. In: M. Iannelli, R. Nagel and S. Piazzera (Eds.), "Functional Analytic Methods for Evolution Equations," Lecture Notes in Mathematics 1855, Springer-Verlag, Berlin, 2004, pp. 401-472.

[19] A. Yagi, Parabolic equations in which the coefficients are generators of infinitely differentiable semigroups II. Funkcial. Ekvac. 33 (1990), 139-150.

[20] A. Yagi, Abstract quasilinear evolution equations of parabolic type in Banach spaces. Bolletino U.M.I. (7) 5 (1991), 341-368.

L. Maniar, Cadi Ayyad University, Faculty of Sciences Semlalia, B.P. 2390, Marrakesh, MOROCCO.

E-mail address: maniar@ucam.ac.ma, lama@fa.uni-tuebingen.de

R. Schnaubelt, Institut für Analysis, Fakultät für Mathematik, Universität Karlsruhe, 76128 Karlsruhe, Germany.

E-mail address: schnaubelt@math.uni-karlsruhe.de 